\title{
Parallel Transport in the Determinant Line Bundle: The Zero Index Case
}

\author{
S. Della Pietra ${ }^{1,2 \star}$ and V. Della Pietra ${ }^{1 \star \star}$ \\ 1 Lyman Laboratory of Physics, Harvard University, Cambridge, MA02138, USA \\ 2 Theory Group, Physics Department, University of Texas, Austin, TX78712, USA
}

\begin{abstract}
For a product family of invertible Weyl operators on a compact manifold $X$, we express parallel transport in the determinant line bundle in terms of the spectral asymmetry of a Dirac operator on $\mathbf{R} \times X$.
\end{abstract}

\section{Introduction}

Let $X$ be a compact spin manifold of even dimension with spin bundle $S=S_{+} \oplus$ $S_{-} \rightarrow X$, and let $E \rightarrow X$ be the hermitian vector bundle over $X$. Let $\bar{S}$ and $\bar{E}$ be the pullbacks of $S$ and $E$ to $\mathbf{R} \times X$ with the induced inner products, and let $\bar{\nabla}^{E}$ be a connection on $\bar{E}$. Thus $\bar{\nabla}^{E}=d_{\mathbf{R}}+\theta+\nabla_{(\cdot)}^{E}$, where $\theta \in \Omega^{1}(\mathbf{R}) \otimes C^{\infty}(X$, End $E)$ and for each $y \in \mathbf{R}, \nabla_{y}^{E}$ is a connection of $E \rightarrow X$. Let $\partial_{y}, y \in \mathbf{R}$, be the Weyl operators $\partial_{y}$ : $L^{2}\left(X, S_{+} \otimes E\right) \mapsto L^{2}\left(X, S_{-} \otimes E\right)$ coupled to the connection $\nabla_{y}^{E}$ and the $(y-$ independent) metric on $X$, and let $\nabla \partial=d_{\mathbf{R}} \partial+[\theta, \partial]$. Let $H$ be the formally self adjoint Dirac operator on $L^{2}(\mathbf{R} \times X, \bar{S} \otimes \bar{E})$ coupled to $\bar{\nabla}^{E}$ and the product metric on $\mathbf{R} \times X$. Thus

$$
H=\left(\begin{array}{cc}
i\left(\frac{\partial}{\partial y}+\theta\left(\frac{\partial}{\partial y}\right)\right) & \partial_{y}^{\dagger} \\
\partial_{y} & -i\left(\frac{\partial}{\partial y}+\theta\left(\frac{\partial}{\partial y}\right)\right)
\end{array}\right) .
$$

Assume that for all $y \in \mathbf{R}, \partial_{y}$ is invertible (so that ind $\partial_{y}=0$ ), and that for $|y|$ large, $\theta=0$ and $d \nabla^{E} / d y=0$. The main result of this paper is the formula

$$
\exp \int_{R} \operatorname{Tr} \partial^{-1} \nabla \partial=\left(\frac{\operatorname{det} \partial_{\infty}^{\dagger} \partial_{\infty}}{\operatorname{det} \partial_{-\infty}^{\dagger} \partial_{-\infty}}\right)^{1 / 2} \exp \pi i(\eta(H)+\operatorname{dim} \operatorname{Ker} H) .
$$

Here $\operatorname{det} \partial_{y}^{\dagger} \partial_{y}$ is the determinant of $\partial_{y}^{\dagger} \partial_{y}$, given formally as the product of the

\footnotetext{
* Supported in party by NSF Grants PHY8605978 and PHY-82-15249 and the Robert A. Welch Foundation

$\star \star$ Supposed in part by NSF Grant PHY-82-15249
} 
eigenvalues of $\partial_{y}^{\dagger} \partial_{y}$, and $\eta(H)$ is a measure of the spectral asymmetry of $H$ as introduced in [4] and given formally by the sum of the signs of the nonzero eigenvalues of $H$. Of course each term in (0.1) requires regularization, and moreover, since the noncompactness of $\mathbf{R} \times X$ means that $H$ does not necessarily have a discrete spectrum, the definition of $\eta$ requires some care. We will deal with these issues by expressing the quantities in (0.1) in terms of operator traces, regulated by the complex powers $\left(\partial_{y}^{\dagger} \partial_{y}\right)^{-z}$ and $\left(H^{2}\right)^{-z}, \operatorname{Re} z \gg 0$. For the determinant this corresponds to $\zeta$-function regularization.

The left-hand side of $(0.1)$ can be interpreted in terms of parallel transport in the determinant line bundle of the family of operators $\left\{\partial_{y}\right\}$, as we explain in Sect. 1 below. From this point of view, formula $(0.1)$ can be considered as a version for nonclosed paths of the holonomy formula of Witten [18] and Bismut and Freed ([6], Theorem 3.17).

Alternately, the left-hand side of $(0.1)$ is related to the phase of the chiral determinant $\operatorname{det} \partial_{-\infty}^{\dagger} \partial_{\infty}$. In this context, a formula similar to (0.1) appears in [1]. We investigate this viewpoint in our paper [2].

The organization of this paper is as follows. In Sect. 1 we state our results and describe how they are related to the geometry of the determinant line bundle. In Sect. 2 we define precisely the determinant det $\partial^{\dagger} \partial$ and the one-form $\operatorname{Tr} \partial^{-1} \nabla \partial$ which appear in (0.1), and in Sect. 3 we define $\eta(H)$. Then in Sect. 4 we obtain a formula for the variation of $\eta(H)$ with respect to $H$. In Sect. 5 we integrate this formula to prove $(0.1)$ and then in Sect. 6 we use this formula to give a proof of the curvature formula of Bismut and Freed.

In Appendix A we investigate the spectral properties of the operators $D^{2}, \partial^{\dagger} \partial$ and $H^{2}$, and we prove an important decay property for the resolvent of $H^{2}$. In Appendix $\mathrm{B}$ we state a generalization of Gilkey's Theorem [3]. In Appendix C we extend some results of Seeley $[15,16]$ and state formulae for local invariants obtained from (the analytic continuation of) $\operatorname{Tr} A(z)$, where $A(z)=\int_{C}(d \lambda / 2 \pi i) \lambda^{-z} R(\lambda)$ for a suitable family of pseudo-differential operators $R(\lambda)$. (Seeley considered the case where $R(\lambda)$ is the resolvent of a elliptic operator.) The results of Appendix B and Appendix C are used in the body of the paper to establish the finiteness of our regularization procedure. In Appendix D we present our notational conventions.

In our subsequent paper [9] we generalize the results of this paper to include families of Weyl operators of possibly non-zero index.

\section{Statement of Results}

In this section we state the main results of this paper and describe how they fit into the framework developed by Bismut and Freed $[6,7]$ for studying the determinant line bundle of a family of Weyl operators.

We work in the special case of the Bismut-Freed setting in which the geometric data have a product structure. The parameter space for our family of operators is a smooth manifold $Y$. For formula (0.1) we will take $Y$ to be the real line $Y=\mathbf{R}$. Let $X$ be a compact spin manifold of even dimension, and let $Z=Y \times X$, which we view as fibered over $Y$ with fiber $X$ and tangent space along the fibers $T^{\text {vert }} Z=Y \times T X$. Put 
a metric on $X$ and the corresponding $y$-independent inner product on $T^{\text {vert }} Z$. Let $\bar{S}_{ \pm} \rightarrow Z$ be the spin bundles associated to $T^{\text {vert }} Z$, so that $\bar{S}_{ \pm}=Y \times S_{ \pm}$, where $S_{ \pm} \rightarrow X$ are the spin bundles on $X$. Let $\bar{E} \rightarrow Z$ be a complex vector bundle over $Y$ which is of the form $\bar{E}=Y \times E$ for $E \rightarrow X$ a vector bundle over $X$. Put a hermitian inner product on $E$ and the corresponding $y$-independent inner product on $\bar{E}$. Let $\nabla^{\bar{E}}$ be a compatible connection on $\bar{E}$. Finally, choose the projection $P: T Z \mapsto T^{\text {vert }} Z=$ $Y \times T X$ of the Bismut-Freed data to be given by the product structure. ${ }^{1}$

The constructions of Bismut and Freed applied to these data now yield a Hilbert bundle $\mathscr{H}=\mathscr{H}_{+} \oplus \mathscr{H}_{-} \rightarrow Y$ with an inner product and connection $\nabla^{\mathscr{H}}$, a bundle map $\partial: \mathscr{H}_{+} \mapsto \mathscr{H}_{-}$given by a Weyl operator $\partial_{y}$ on each fiber, and a determinant line bundle $\mathscr{L} \rightarrow Y$ with an inner product and compatible connection $\nabla^{\mathscr{L}}$. If the ordinary index of the operators $\partial_{y}$ is zero, the bundle $\mathscr{L}$ has a canonical section $s$ which is nonzero exactly at those $y$ for which $\partial_{y}$ is invertible.

In our case we can describe these structures explicitly. Using the product structure $\bar{E}=Y \times E$, write

$$
\nabla^{\bar{E}}=d_{Y}+\theta+\nabla_{(\cdot)}^{E}
$$

where $\theta \in \Omega^{1}(Y) \otimes C^{\infty}(X$, End $E)$, and for each $y \in Y, \nabla_{y}^{E}$ is a connection on $E \rightarrow X$. The Hilbert bundle $\mathscr{H}$ is trivial with $\mathscr{H}_{ \pm}=Y \times L^{2}\left(X, S_{ \pm} \otimes E\right)$. The inner product on $\mathscr{H}$ is $y$-independent and given by the inner product on $L^{2}(X, S \otimes E)$. The connection $\nabla^{\mathscr{H}}$ on $\mathscr{H}$ is given by $\nabla^{\mathscr{H}}=d_{Y}+\theta$. The operator $\partial_{y}$ is identified with the Weyl operator $\partial_{y}: L^{2}\left(X, S_{+} \otimes E\right) \rightarrow L^{2}\left(X, S_{-} \otimes E\right)$ coupled to the metric on $X$ and the connection $\nabla_{y}^{E}$. The covariant derivative of $\partial$ as a section of $\operatorname{Hom}\left(\mathscr{H}_{+}, \mathscr{H}_{-}\right)$ is given by $\nabla \partial=d_{Y} \partial+[\theta, \partial]$.

Finally, in the case that the operators $\partial_{y}$ have index zero, the inner product and connection on $\mathscr{L}$ are determined at those points where the canonical section $s$ does not vanish by the equations

$$
\begin{gathered}
\nabla^{\mathscr{L}} s=s w, \quad\|s\|_{\mathscr{L}}^{2}=\operatorname{det} \partial^{\dagger} \partial, \\
\omega=\{\text { f.p.a.z }=0\} \operatorname{Tr}\left(\partial^{\dagger} \partial\right)^{-z-1} \partial^{\dagger} \nabla^{\operatorname{Hom}\left(\mathscr{C}_{+}, \mathscr{H}_{-}\right)} \partial, \\
\operatorname{det} \partial^{\dagger} \partial=\exp -\lim _{z \rightarrow 0} \frac{d}{d z} \operatorname{Tr}\left(\partial^{\dagger} \partial\right)^{-z} .
\end{gathered}
$$

Here the complex powers $\left(\partial^{\dagger} \partial\right)^{-z-1}$ are defined for $z \in \mathbf{C}, \operatorname{Re} z \gg 0$ by contour integration, and the notation $\lim _{z \rightarrow 0}$ (respectively $\{$ f.p.a.z $=0\}$ ) is understood as the value at $z=0$ (respectively the finite part at $z=0$ ) of the meromorphic continuation of a function which is analytic for $\operatorname{Re} z \gg 0$. We give the precise interpretation of (1.3) and (1.4) in Sect. 2.

We henceforth assume

1. For all $y \in Y, \operatorname{Ker} \partial=0$ and $\operatorname{Ker} \partial^{\dagger}=0$.

Thus, in particular, index $\partial_{y}=0$. Condition 1 is equivalent to the condition that $\partial_{y}$ is

1 Note that for arbitrary geometric data as in [6,7], the space $Z$ and the bundles $T^{\text {vert }} Z, \bar{S}, \bar{E}$ are always products locally over $Y$. However, our form for the inner product on $T^{\text {vert }} Z$ and the projection $P$ do not hold in general, even locally 
invertible for all $y \in Y$, and also to the condition that the canonical section $s$ of $\mathscr{L}$ is everywhere non-vanishing.

For our first result, we will verify in our special case the curvature formula of Bismut and Freed ([6], Theorem 3.5).

Theorem (1.5). Assuming condition 1, the curvature of $\nabla^{\mathscr{L}}$ is given by the two-form on $Y$

$$
d \omega=2 \pi i\left[\int_{X} \hat{A}\left(\mathscr{R}_{g}\right) \operatorname{ch}\left(\mathscr{F}_{\nabla \bar{E}}\right)\right]_{\{\text {two forms }\}} .
$$

Here $g$ is the ( $Y$-independent) metric on $X, \mathscr{R}_{g}$ is the curvature of the Levi-Civita connection of $g$, and $\mathscr{F}_{\nabla \bar{E}}$ is the curvature of the connection $\nabla^{\bar{E}}$ on $\bar{E}$. $\hat{A}$ and ch are the polynomials

$$
\hat{A}(\mathscr{R})=\sqrt{\operatorname{det}\left(\frac{\mathscr{R} / 4 \pi}{\sinh \mathscr{R} / 4 \pi}\right)}, \quad \operatorname{ch}(\mathscr{F})=\operatorname{trexp} i \mathscr{F} / 2 \pi .
$$

Next suppose $Y=\mathbf{R}$. Give $\mathbf{R}$ the standard translation invariant metric $d y \otimes d y$ and give $Z=\mathbf{R} \times X$ the product metric. The bundle $\bar{S} \rightarrow Z$ is then identified with a spin bundle of $Z$. Let $H$ be the formally self-adjoint Dirac operator on $L^{2}(Z, \bar{S} \otimes \bar{E})$ coupled to the metric on $Z$ and the connection $\nabla^{\bar{E}}$ on $\bar{E}$. In terms of the product structure $\bar{S} \otimes \bar{E}=Y \times(S \otimes E)$,

$$
H=i \Gamma\left(\frac{\partial}{\partial y}+\theta\left(\frac{\partial}{\partial y}\right)\right)+D_{(\cdot)}=\left(\begin{array}{cc}
i\left(\frac{\partial}{\partial y}+\theta\left(\frac{\partial}{\partial y}\right)\right) & \partial_{y}^{\dagger} \\
\partial_{y} & -i\left(\frac{\partial}{\partial y}+\theta\left(\frac{\partial}{\partial y}\right)\right)
\end{array}\right)
$$

where $\Gamma$ is the endomorphism of $S$ with $\Gamma= \pm 1$ on $S_{ \pm}$.

In addition to condition 1 assume

2. For $|y|$ large, $\theta=0$ and $d \nabla^{E} / d y=0$.

Thus for $|y|$ large the geometric data is independent of $y$. In particular for $|y|$ large, $d_{Y} \partial=0, \nabla \partial=0, \omega=0$, and $H$ is invariant under translation in the $\mathbf{R}$ direction.

Define

$$
\begin{aligned}
& \eta(H)=\lim _{z \rightarrow 0} \lim \operatorname{Tr} \phi H\left(H^{2}\right)^{-z-1 / 2}, \\
& \xi(H)=\frac{1}{2}(\eta(H)+\operatorname{dim} \operatorname{Ker} H) .
\end{aligned}
$$

Here $\phi$ is a nonnegative smooth function on $\mathbf{R}$ of compact support acting as a multiplication operator on $L^{2}(\mathbf{R} \times X, \bar{S} \otimes \bar{E})$, and the limit $\phi \rightarrow 1$ is taken through a sequence of such $\phi$ increasing pointwise to the constant function 1 . We introduce these cut-off functions in order to obtain trace class operators. The complex powers of $H^{2}$ are defined by contour integration, and $\lim _{z \rightarrow 0}$ is understood in terms of analytic continuation. We will give the precise interpretation of (1.8) and (1.9) in Sect. 3.

Our main result is 
Theorem (1.10). Assuming conditions 1 and 2 , parallel transport $\tau_{+\infty,-\infty}$ for $\nabla^{\mathscr{L}}$ from $-\infty$ to $+\infty$ is given by

$$
\begin{aligned}
\tau_{+\infty,-\infty} s_{-\infty} & =s_{+\infty} \exp -\int_{\mathbf{R}} \omega, \\
\exp \int_{\mathbf{R}} \omega & =\left(\frac{\operatorname{det} \partial_{\infty}^{\dagger} \partial_{\infty}}{\operatorname{det} \partial_{-\infty}^{\dagger} \partial_{-\infty}}\right)^{1 / 2} \exp 2 \pi i \xi(H) .
\end{aligned}
$$

Of course (1.11) follows immediately from (1.2) which identifies $\omega$ as the connection one-form relative to the canonical section $s$, so that the non-trivial statement is (1.12).

Theorem (1.10) is an extension, under our additional assumptions of the formula of Bismut and Freed ([6], Theorem 3.17) for the holonomy of $\nabla^{\mathscr{L}}$. In fact, Bismut and Freed showed that quite generally the holonomy of $\nabla^{\mathscr{L}}$ around a closed loop $\gamma$ in $Y$ is given by multiplication by $\exp -2 \pi i \xi(H)$, where $H$ acts on spinors over the compact manifold $\gamma \times X$, and $\eta(H)=\lim _{z \rightarrow 0} \sum \operatorname{sign}(\lambda)|\lambda|^{-z}$ with the sum taken over the nonzero eigenvalues of $H$. Thus, in our situation we might expect that

$$
\exp i \int_{\mathbf{R}} \operatorname{Im} \omega=\exp 2 \pi i \xi(H) .
$$

On the other hand, since $\nabla^{\mathscr{L}}$ is compatible with the inner product $\|\cdot\|_{\mathscr{L}}$, it follows from (1.2) that $\operatorname{Re} \omega=\frac{1}{2} d \ln \operatorname{det} \partial^{\dagger} \partial$, and so

$$
\exp \int_{\mathbf{R}} \operatorname{Re} \omega=\left(\frac{\operatorname{det} \partial_{\infty}^{\dagger} \partial_{\infty}}{\operatorname{det} \partial_{-\infty}^{\dagger} \partial_{-\infty}}\right)^{1 / 2}
$$

Theorem (1.10) is obtained by combing (1.13) and (1.14). Of course, on the noncompact manifold $Z=\mathbf{R} \times X$, there is no reason for the spectrum of $H$ to be discrete, so we have defined $\eta(H)$ by the alternate expression (1.8).

\section{The Determinant and $\omega$}

In this section we explain the formulas (1.3) and (1.4) for the determinant and the one-form $\omega$. We allow the parameter space $Y$ to be an arbitrary smooth manifold, and assume that condition 1 of Sect. 1 is satisfied.

Let $D: \mathscr{H} \mapsto \mathscr{H}$ be the family of formally self-adjoint Dirac operators corresponding to $\partial$, so that $D_{y}$ is the operator on $L^{2}(X, S \otimes E)$ which in the decomposition $S=S_{+} \otimes S_{-}$is given by $D_{y}=\left(\begin{array}{ll} & \partial_{y}^{\dagger} \\ \partial_{y} & \end{array}\right)$. For convenience we will work with $D$ rather than $\partial$. By condition $1, D_{y}$ is invertible for all $y$.

For $y \in Y$ and $A$ a first order differential operator acting on sections of $S \otimes E$ define $^{2}$

2 We introduce here a notational convention, to be used in the remainder of this paper, of marking operator valued expressions with hat , and denoting their $L^{2}$ traces by the same symbol without a hat 


$$
\begin{aligned}
& \hat{\zeta}\left(D_{y}^{2}\right)(z)=\left(D_{y}^{2}\right)^{-z}=\int_{\mathscr{Q}} \frac{d \lambda}{2 \pi i} \lambda^{-z}\left(D_{y}^{2}-\lambda\right)^{-1}, \quad \operatorname{Re} z>0 \\
& \zeta\left(D_{y}^{2}\right)(z)=\operatorname{Tr} \hat{\zeta}\left(D_{y}^{2}\right)(z), \quad \quad \operatorname{Re} z>\frac{1}{2} \operatorname{dim} X \\
& \operatorname{det} D_{y}^{2}=\exp -\lim _{z \rightarrow 0} \frac{d}{d z} \zeta\left(D_{y}^{2}\right)(z) \\
& \hat{\tau}\left(D_{y}, A\right)(z)=\frac{1}{2}\left(D_{y}^{2}\right)^{-z-1} D_{y} A=-\frac{1}{2} \int_{\mathscr{\zeta}} \frac{d \lambda}{2 \pi i} \lambda^{-z-1}\left(D_{y}^{2}-\lambda\right)^{-1} D_{y} A, \quad \operatorname{Re} z>0, \\
& \tau\left(D_{y}, A\right)(z)=\operatorname{Tr} \hat{\tau}\left(D_{y}, A\right)(z) \quad \sigma\left(D_{y}, A\right)(z)=\operatorname{Tr}_{s} \hat{\tau}\left(D_{y}, A\right)(z), \quad \operatorname{Re} z>\frac{1}{2} \operatorname{dim} X
\end{aligned}
$$

Here $\mathscr{C}$ is the oriented curve in $\mathbf{C}$ with runs from $-\infty$ to $-\delta, \delta>0$, directly above the negative real axis, then clockwise around the circle $|\lambda|=\delta$, and then from $-\delta$ to $-\infty$ directly below the negative real axis. $\delta$ is chosen sufficiently small so that the disk $|\lambda|<2 \delta$ is disjoint from the spectrum of $D_{y}^{2}$. This is possible since $D_{y}^{2}$ is elliptic, self-adjoint, and by assumption invertible so that its spectrum consists of isolated points in $(0, \infty)$. (See Proposition A.1 of Appendix A.)

The complex powers $\lambda^{-z}$ for $\lambda \in \mathbf{C} \backslash(-\infty, 0], z \in \mathbf{C}$, are defined in terms of the branch of the logarithm with cut along the negative real axis and $\log (1)=0 . \operatorname{Tr}$ denotes the $L^{2}$ operator trace and $\operatorname{Tr}_{s}$ denotes the super-trace, defined by $\operatorname{Tr}_{s}=$ $\operatorname{Tr} \circ \Gamma$. Finally, the notation $\lim$ is understood as the value at $z=0$ of the analytic continuation of a function which is analytic for $\operatorname{Re} z \gg 0$.

Note that the definition (1.3) of $\omega$ is equivalent to

$$
\omega=\{\text { f.p.a.z }=0\}(\tau(D, \nabla D)(z)+\sigma(D, \nabla D)(z)),
$$

and this gives the decomposition of $\omega$ into its real and imaginary part.

The main result of this section is

\section{Proposition (2.8).}

1. The definitions (2.1)-(2.5) make sense, and $\zeta\left(D_{y}^{2}\right)(z), \tau\left(D_{y}, A\right)(z), \sigma\left(D_{y}, A\right)(z)$ extend to meromorphic functions of $z$ for $\operatorname{Re} z>-1$ whose only singularities are possible simple poles at half-integer values of $z$.

2. $\{$ f.p.a. $z=0\} 4 \tau(D, \nabla D)(z)=d \ln \operatorname{det} D^{2}$.

3. $\sigma(D, d D)(z)$ and $\sigma(D,[\theta, D])(z)$ are analytic for $\operatorname{Re} z>-\frac{1}{2}$. Moreover

$$
\lim _{z \rightarrow 0} \sigma(D,[\theta, D])(z)=-\int_{X} \hat{A}\left(\mathscr{R}_{g}\right) \operatorname{tr} \theta \exp i \mathscr{F}_{\nabla^{E}} / 2 \pi .
$$

Here $g$ is the metric on $X, \mathscr{R}_{g}$ is the curvature of the Levi-Civita connection for $g$, and $\mathscr{F}_{\nabla^{E}}$ is the curvature of the connection $\nabla^{E}$ on $E$. $\hat{A}$ is the polynomial given in (1.6). Formula (2.8.2) appears in the physics literature in the context of the "covariant anomaly."

We remark that if det $\partial^{\dagger} \partial$ are defined in analogy with $\operatorname{det} D^{2}$, then

$$
\operatorname{det} D^{2}=\left(\operatorname{det} \partial^{\dagger} \partial\right)\left(\operatorname{det} \partial \partial^{\dagger}\right), \quad \operatorname{det} \partial^{\dagger} \partial=\operatorname{det} \partial \partial^{\dagger},
$$


and so (2.7) and (2.8.2) imply

$$
\omega=\frac{1}{2} d \ln \operatorname{det} \partial^{\dagger} \partial+\sigma(D, \nabla D) .
$$

Statement (2.8.1) follows from the general pseudo-differential operator methods of [15] (see Appendix C). Statement (2.8.2) is an immediate consequence of the following lemma, whose proof is an easy calculation (see [8]).

Lemma (2.11). $d\left(\zeta\left(D^{2}\right)(z)\right)=-4 z \tau(D, \nabla D)(z)$.

It remains to prove (2.8.3). Now the general methods used to prove (2.8.1) express the residues of the poles as well as the value at $z=0$ of the kernel of $\sigma(D, A)(z)$ in terms of certain universal polynomials in the components of $g$, $\operatorname{det} g$, the covariants derivatives of $\mathscr{R}_{g}, \mathscr{F}_{\nabla^{E}}$ and the complete symbol of $A$. (See Appendix C, as well as Lemma (3.7) of the next section.) To prove (2.8.3) we must in principle calculate these polynomials. This is possible using invariance theory and Gilkey's Theorem [3] (see Appendix B) because of the special properties of Dirac operators.

Specifically, for $v \in \Omega^{1}(X$, End $E)$, let $\not b$ denote the bundle endomorphism of $S \otimes E$ determined by $v$ and Clifford multiplication $T^{*} X \mapsto$ End $S$. Statement (2.8.3) is an immediate consequence of the following lemma.

\section{Lemma (2.12).}

1. $\operatorname{Res}_{z=0} \operatorname{tr}_{s} \hat{\tau}(D, \psi)(z ; x, x)|d x|$ is an exact differential form on $X$.

2. $\operatorname{Res}_{z=k / 2} \operatorname{tr}_{s} \hat{\imath}(D, \hat{k})(z ; x, x)|d x|=0$ for integer $k, k>0$.

3. $\lim _{z \rightarrow 0} \operatorname{tr}_{s} \hat{\tau}(D,[\theta, D])(z ; x, x)|d x|=-\hat{A}\left(\mathscr{R}_{g}\right) \operatorname{tr} \theta \exp i \mathscr{F}_{\nabla^{E}} / 2 \pi$.

Proof. In the language of Appendix B, the assignment

$$
\left(g, \nabla^{E}, v\right) \mapsto \operatorname{Res}_{z=0} \operatorname{tr}_{s} \hat{\tau}(D, \phi)(z ; x, x)|d x|
$$

defines a weight zero, regular, form-valued invariant of the metric $g$ on $X$, the connection $\nabla^{E}$, and the endomorphism valued one-form $v$. (Note that (2.13) defines a differential form valued invariant, as opposed to just a measure, since $D$ and $v$ are even under change of orientation of $X$ while $\operatorname{Tr}_{s}$ is odd.) Hence, by the Gilkey Theorem B.1, (2.13) is in the ring of invariants generated by $\operatorname{tr}\left(\mathscr{R}_{g}^{j}\right)$, and $\operatorname{tr} m\left(\mathscr{F}_{\nabla^{E}}, v, d_{\nabla} v\right)$. Here $d_{\nabla}$ is the covariant exterior derivative determined by the connections on $T X$ and $E$. We view $\mathscr{F}_{\nabla^{E}}, v$, and $d_{\nabla} v$ as elements of the ring $\Omega *(X$, End $E)$, and $\mathscr{R}_{g}$ as an element of the ring $\Omega *(X$, End $T X) . m(\cdots)$ is a monomial in 4 variables and $j$ is a positive integer, Since $\operatorname{Res}_{z=0} \operatorname{tr}_{s} \sigma(D, \phi)(z ; x, x)|d x|$ is linear in $v$ and a differential form of even degree, it is expressible as a linear combination of products of terms of the form $\operatorname{tr}\left(\mathscr{R}_{g}^{j}\right), \operatorname{tr}\left(\mathscr{F}_{\nabla^{E}}^{j}\right)$, and $\operatorname{tr} d_{\nabla} v \mathscr{F}{ }_{\nabla} E$, and one term of the latter type must occur in each product. Since terms of the first two types are closed and terms of the last type are exact by the Bianchi identities for $\mathscr{F}_{\nabla}{ }^{E}$ and $\mathscr{R}_{g}$, it follows that $\operatorname{Res}_{z=0} \operatorname{tr}_{s} \hat{\tau}(D, \phi)(z ; x, x)|d x|$ is exact. This proves (2.12.1)

(2.12.2) follows similarly, since for $k>0$ the residues at $z=k / 2$ are also differential form valued invariants, but now of positive weight. They thus vanish by the easy part of the Gilkey Theorem.

Finally, to prove $(2.12 .3)$ observe that $\lim _{z \rightarrow 0} \operatorname{tr}_{s} \hat{\tau}(D,[\nabla, D])(z ; x, x)|d x|$ defines a weight zero form valued invariant of $g, \nabla^{E}$, and the (weight zero) endomorphism $\theta$, 
and so by Gilkey's Theorem it is in the ring generated by $\operatorname{tr}\left(\mathscr{R}_{g}^{j}\right)$ and $\operatorname{tr} m\left(\theta, \mathscr{F}_{\nabla}\right)$. The actual formula given in (2.12.3) can then be calculated in a straightforward but tedious fashion using the explicit formulae of Corollary (C.8).

\section{The Eta Invariant}

In this section we explain the formulas (1.8)-(1.9) for the $\eta$-invariant of $H$. We now let $Y=\mathbf{R}$ and assume that the geometric data satisfy conditions 1 and 2 of Sect. 1 .

Because of the non-compactness of $\mathbf{R} \times X$, the spectrum of $H$ is not discrete, and so we cannot use the usual definition of $\eta(H)$ in terms of eigenvalues. Fortunately, however, conditions 1 and 2 imply that the essential spectrum and resolvent of $H^{2}$ are well enough behaved for us to define the complex powers $\left(H^{2}\right)^{-z}$, and we will use these to define $\eta(H)$.

Define

$$
\begin{aligned}
\hat{\eta}^{\delta}(H)(z) & =H\left(H^{2}\right)^{-z-1 / 2}=-\int_{\mathscr{C}} \frac{d \lambda}{2 \pi i} \lambda^{-z-1 / 2} H\left(H^{2}-\lambda\right)^{-1}, \quad \operatorname{Re} z>0 \\
\eta_{\phi}^{\delta}(H)(z) & =\operatorname{Tr} \phi \hat{\eta}^{\delta}(H)(z), \quad \operatorname{Re} z>\operatorname{dim} Z+1, \\
\eta^{\delta}(H)(z) & =\lim _{n \rightarrow \infty} \eta_{\phi_{n}}^{\delta}(H)(z), \\
\eta(H) & =\lim _{z \rightarrow 0} \eta^{\delta}(H)(z)+\sum_{0<|\lambda|<\delta} \operatorname{sign}(\lambda) \operatorname{Tr} P_{\lambda}, \\
\xi(H) & =\frac{1}{2}(\eta(H)+\operatorname{dim} \operatorname{Ker} H) .
\end{aligned}
$$

Here $\mathscr{C}$ is a contour in $\mathbf{C}$ as in the previous section. $\delta$ is chosen sufficiently small so that the disk $|\lambda|<2 \delta$ is disjoint from the spectra of $D_{ \pm \infty}^{2}$ and intersects the spectrum of $\mathrm{H}^{2}$ at most in a finite set of isolated eigenvalues of finite multiplicity. This is possible since the essential spectrum of $H^{2}$ is contained in $\left[\lambda_{0}, \infty\right)$, where $\lambda_{0}$ is the lower bound of the spectra of $D_{ \pm \infty}^{2}$. (See Proposition (A.2) of Appendix A.) By condition $1, D_{ \pm \infty}^{2}$ are invertible, and so $\lambda_{0}>0$.

$\phi$ is a smooth nonnegative real-valued function on $\mathbf{R}$ of compact support, and $\left\{\phi_{n}\right\}$ are an increasing sequence of such functions which converge pointwise to the constant function 1. In (3.2) and (3.3) we interpret $\phi$ and $\phi_{n}$ as a multiplication operators on $L^{2}(\mathbf{R} \times X, \bar{S} \otimes \bar{E})$. The reason for introducing these cut-off functions is that in general the operator $\hat{\eta}^{\delta}(H)(z)$ is not trace class. ${ }^{3}$

As in the previous section, $\lim$ is interpreted in terms of analytic continuation.

Finally $P_{\lambda}$ is the orthogonal projection onto the finite dimensional eigenspace corresponding to $\lambda$, and $\operatorname{sign}(\lambda)$ equals -1 if $\lambda<0$ and +1 otherwise.

Our goal in this section is to prove

\section{Proposition (3.6).}

1. The definitions (3.1) and (3.2) make sense, and $\eta_{\phi}^{\delta}(H)(z)$ extends to an analytic function of $z$ for $\operatorname{Re} z>-\frac{1}{2}$.

3 Similar cut-off functions were introduced by J. Lott [12] in defining the $\eta$-invariant for Dirac operators on $R^{2 m+1}$ 
2. In definition (3.3), the $\eta_{\phi_{n}}^{\delta}(H)(z)$ converge uniformly in $z$ on compact sets for $\operatorname{Re} z>$ $-\frac{1}{2}$.

3. $\eta^{\delta}(H)(z)$ is analytic in $z$ for $\operatorname{Re} z>-\frac{1}{2}$ and depends smoothly on $H$ and $z$ for sufficiently small variations of $H . \eta(H) \bmod 1$ and $\xi(H) \bmod 1$ are independent of $\delta$ and depend smoothly on $H$.

We begin with

\section{Lemma (3.7).}

1. For $\operatorname{Re} z>0, \hat{\eta}^{\delta}(H)(z)$ is a bounded operator on $L^{2}(\mathbf{R} \times X, \bar{S} \otimes \bar{E})$.

2. For $\operatorname{Re} z>\frac{1}{2} \operatorname{dim} Z, \hat{\eta}^{\delta}(H)(z)$ has a continuous kernel $\hat{\eta}^{\delta}(H)(z ; y, x, y, x) d y|d x|$ which is analytic in $z$.

3. $\operatorname{tr} \hat{\eta}^{\delta}(H)(z ; y, x ; y, x)$ extends to a meromorphic function of $z$ for $\operatorname{Re} z>-1$ which is continuous in $y, x$, smooth in $H$ for small variations of $H$, and has possible poles only at half-integer values of $z$. These poles are simple and their residues are given by universal polynomials in the components of $\bar{g},(\operatorname{det} \bar{g})^{-1}$, and the covariant derivatives of $\mathscr{F}_{\nabla \bar{E}}$ and $\mathscr{R}_{\bar{g}}$.

4. For $\operatorname{Re} z>\operatorname{dim} Z+\frac{1}{2}$ and $\phi$ with compact support, $\phi \hat{\eta}^{\delta}(H)(z)$ is trace class with trace given by $\int_{Z} \operatorname{tr} \phi(y) \hat{\eta}^{\delta}(H)(z ; y, x ; y, x) d y|d x|$.

Here $\mathscr{F}_{\nabla^{E}}$ is the curvature of the connection $\nabla^{\bar{E}}$ on $\bar{E}, \bar{g}$ is the metric on $Z$, and $\mathscr{R}_{\bar{g}}$ is the curvature of the Levi-Cevita connection for $\bar{g}$. Note that (3.7.3) allows a pole at $z=0$; we will eliminate this possiblity in the next Proposition. We remark that (3.7.4) relies on conditions 1 and 2 which imply that the resolvent of $H^{2}$ has suitable decay (see Proposition (A.6) of Appendix A).

Proof. This lemma is essentially standard, although some care is required to deal with the non-compactness. The usual methods for proving (3.7.1) remain valid because we are using a translation invariant $L^{2}$ norm, and we are assuming that $H$ becomes translation invariant for $|y|>1$. Similarly, a kernel of $\hat{\eta}^{\delta}(H)(z)$ can be constructed by the standard pseudo-differential operator techniques, and then the analyticity and locality properties (3.7.2) and (3.7.3) follow as usual (see also Appendix C).

The only new difficulty is in showing that for $\operatorname{Re} z$ sufficiently large, $\phi \hat{\eta}^{\delta}(H)(z)$ is trace class. For this we use the decay estimates of Proposition A.6 as follows. Set $k=(\operatorname{dim} Z+1) / 2$ and suppose $\operatorname{Re} z>\operatorname{dim} Z+\frac{1}{2}$. Integration by parts in $\lambda$ shows that

$$
\phi \hat{\eta}^{\delta}(H)(z)=\text { const } \int_{\mathscr{C}} \frac{d \lambda}{2 \pi i} \lambda^{-z-1 / 2+2 k} \phi H\left(H^{2}-\lambda\right)^{-1-2 k} .
$$

We will show that the integrand $\phi H\left(H^{2}-\lambda\right)^{-1-2 k}$ in (3.8) is trace class with trace norm bounded uniformly in $\lambda$ for $\lambda \in \mathscr{C}$. Then clearly $\phi \hat{\eta}^{\delta}(H)(z)$ is trace class.

For $\rho>0$ define continuous kernels

$$
\begin{aligned}
& K_{1}(\lambda)\left(y, x, y^{\prime}, x^{\prime}\right)=\phi(y)\left[H\left(H^{2}-\lambda\right)^{-k-1}\right]\left(y, x, y^{\prime}, x^{\prime}\right) e^{\rho\left|y^{\prime}\right| / 2}, \\
& K_{2}(\lambda)\left(y, x ; y^{\prime}, x^{\prime}\right)=e^{-\rho|y| / 2}\left(H^{2}-\lambda\right)^{-k}\left(y, x ; y^{\prime}, x^{\prime}\right) .
\end{aligned}
$$

By Proposition (A.6) we can choose $\rho$ sufficiently small such that for $\left|y-y^{\prime}\right|>1$ and 
$\lambda \in \mathscr{C}$,

$$
\begin{aligned}
& \left|K_{1}(\lambda)\left(y, x ; y^{\prime}, x^{\prime}\right)\right|<\mathrm{const} \phi(y) e^{-\rho\left|y-y^{\prime}\right|} e^{\rho \mid y^{\prime} / 2} \\
& \left|K_{2}(\lambda)\left(y, x ; y^{\prime}, x^{\prime}\right)\right|<\mathrm{const} e^{-\rho|y| / 2} e^{-\rho\left|y-y^{\prime}\right|}
\end{aligned}
$$

Thus, for such a $\rho$, the kernels $\left|K_{1}(\lambda)\left(y, x ; y^{\prime}, x^{\prime}\right)\right|$ and $\left|K_{2}(\lambda)\left(y, x ; y^{\prime}, x^{\prime}\right)\right|$ are square integrable over $Z \times Z$ and they define Hilbert-Schmidt operators $K_{1}(\lambda)$ and $K_{2}(\lambda)$ on $L^{2}(Z, \bar{S} \otimes \bar{E})$ with Hilbert-Schmidt norms bounded uniformly in $\lambda$ for $\lambda \in \mathscr{C}$. It follows that $\phi H\left(H^{2}-\lambda\right)^{-2 k-1}=K_{1}(\lambda) K_{2}(\lambda)$ is trace class with trace norm bounded uniformly in $\lambda$ for $\lambda \in \mathscr{C}$.

As in the previous section, the general pseudo-differential operator techniques used to prove (3.7.3) leave open the possibility that $\eta_{\phi}(H)(z)$ has poles at half-inter values of $z$. However, again the special properties of Dirac operators imply

\section{Lemma (3.11).}

$\operatorname{Res}_{z=k / 2} \operatorname{tr} \hat{\eta}^{\delta}(H)(z ; y, x, y, x) d y|d x|=0$ for integer $k, k \geqq 0$.

The lemma can be proved using Gilkey's Theorem, as in the proof of (2.12) above.

Statement (3.6.1) of Proposition (3.6) now follows immediately from Lemmas (3.7) and (3.11). We next turn to the proof of statement (3.6.2). Let $H_{ \pm \infty}$ be the translation invariant operators on $L^{2}(Z, \bar{S} \otimes \bar{E})$ defined by

$$
H_{ \pm \infty}=i \Gamma \frac{\partial}{\partial y}+D_{ \pm \infty} .
$$

Thus $H_{\infty}$ agrees with $H$ for $y>1$, while $H_{-\infty}$ agrees with $H$ for $y<-1$. The idea is to estimate the behavior of $\eta_{\phi}^{\delta}(H)(z)$ as $\phi \rightarrow 1$ by comparing $\eta_{\phi}^{\delta}(H)(z)$ with $\eta_{\phi}^{\delta}\left(H_{ \pm \infty}\right)(z)$. The next lemma shows that these latter quantities vanish.

Lemma (3.13). Suppose that $H$ is invariant under translations of $Y$. Then Ker $H=\{0\}$ and

$$
\eta_{\phi}^{\delta}(H)(z)=0
$$

Since we are assuming that $\theta$ vanishes for large $|y|$, the hypothesis is equivalent to the conditions $\theta=0$ and $D_{y}=D_{ \pm \infty}$ for all $y$. The lemma can be proved using the explicit expressions

$$
\begin{gathered}
H=i \Gamma \frac{\partial}{\partial y}+D, \quad H^{2}=-\frac{\partial^{2}}{\partial y^{2}}+D^{2} \\
\left(H^{2}-\lambda\right)^{-1}\left(y, x ; y^{\prime}, x^{\prime}\right)=\int_{-\infty}^{\infty} \frac{d E}{2 \pi} e^{i\left(y-y^{\prime}\right) E}\left(D^{2}+E^{2}-\lambda\right)^{-1}\left(x, x^{\prime}\right),
\end{gathered}
$$

where $D=D_{\infty}$ is independent of $y$ and (3.15) is valid for $(y, x) \neq\left(y^{\prime}, x^{\prime}\right), \lambda \in \mathscr{C}$. Note that by assumption $D^{2}$ is invertible, and thus strictly positive.

Now define $\Delta_{ \pm} \hat{\eta}^{\delta}(z)=\hat{\eta}^{\delta}(H)(z)-\hat{\eta}^{\delta}\left(H_{ \pm \infty}\right)(z)$. For $F \subset \mathbf{C}$, let $\rho_{ \pm}(F)$ be the square root of the distance from $F$ to the spectrum of $D_{ \pm}^{2}$. As a consequence of the decay estimates of Proposition (A.6), we have 


\section{Lemma (3.16).}

1. For $y>1, \operatorname{tr} \Delta_{+} \hat{\eta}^{\delta}(z ; y, x, y, x)$ extends to an entire analytic function of $z$.

2. For any compact set $K \subset \mathbf{C}$ there exists a constant $c$ such that for $z \in K$ and $y>1$,

$$
\left|\operatorname{tr} \Delta_{+} \hat{\eta}^{\delta}(z ; y, x, y, x)\right|<c e^{-\rho+(\mathscr{C})|y|} .
$$

These statements hold under the simultaneous replacement of $y>1$ by $y<-1$, $\Delta_{+}$by $\Delta_{-}$, and $\rho_{+}$by $\rho_{-}$.

We omit the proof since we will be proving more delicate decay estimates in Lemma (4.11) below.

Given the previous lemmas, we can now complete the proof of Proposition (3.6).

Proof of (3.6.2): We can assume that $\phi_{n}(y)=1$ for $|y|<1$. Then by Proposition (3.7.4) and (3.13), for $\operatorname{Re} z$ large,

$$
\begin{aligned}
\eta_{\phi}^{\delta}(H)(z)= & \int_{|y|<1} d y \int_{X}|d x| \operatorname{tr} \hat{\eta}^{\delta}(H)(z ; y, x ; y, x) \\
& +\int_{y>1} d y \phi_{n}(y) \int_{X}|d x| \operatorname{tr} \Delta_{+} \hat{\eta}^{\delta}(z ; y, x, y, x) \\
& +\int_{y<-1} d y \phi_{n}(y) \int_{X}|d x| \operatorname{tr} \Delta_{-} \hat{\eta}^{\delta}(z ; y, x, y, x) .
\end{aligned}
$$

By Propositions (3.7.3) and (3.11), the first term on the right-hand side of (3.17) is analytic in $z$ for $\operatorname{Re} z>-\frac{1}{2}$, while by Lemma (3.16) the last two terms are entire analytic functions of $z$ which converge uniformly on compact sets of $\mathbf{C}$ as the $\phi_{n}$ increase to the constant function 1.

Proof of (3.6.3): It is easy to see that for any $\delta$,

$$
\xi(H)=\frac{1}{2} \eta^{\delta}(H)(0)+\frac{1}{2} \operatorname{Tr} P_{|\lambda|<\delta} \bmod 1,
$$

where $P_{|\lambda|<\delta}$ is the orthogonal projection onto the eigenspaces of $H$ with $|\lambda|<\delta$. Now for a smooth family $\left\{H^{t}\right\}, t \in \mathbf{R}$ with $H^{0}=H, \delta$ can be chosen so that for sufficiently small $|t|$, the spectrum of $H^{t}$ is disjoint from the circle $|\lambda|=\delta$. Then $\eta^{\delta}\left(H^{t}\right)(z)$ depends smoothly on $t$ by Proposition (3.7.3), while $\operatorname{Tr} P_{|\lambda|<\delta}\left(H^{t}\right)$ is constant.

\section{The Variation of $\eta(H)$}

In this section we investigate the dependence of $\eta(H)$ on the connection $\nabla^{\bar{E}}$. As in the previous section we assume $Y=\mathbf{R}$.

If $Z$ were an odd dimension compact manifold without boundary, then the first order variation of $\eta(H)$ would be given by a local expression in terms of the curvatures and variations of the connection $\nabla^{\bar{E}}$ and the Levi-Civita connection (see [4]). In our case, because of the non-compactness of $Z=\mathbf{R} \times X$, there is an additional contribution arising from the "boundary" at infinity.

Let $T=\mathbf{R}$ and let $\left\{\bar{\nabla}^{t}\right\}, t \in T$, be a smooth family of connections on $\bar{E} \rightarrow Z$ parametrized by $T$, such that for all $t \in T$ conditions 1 and 2 of Sect. 1 hold. From the constructions of Sect. 1 we obtain a two parameter family $\left\{\nabla_{y}^{t}\right\},(t, y) \in T \times Y$, of connections on $E \rightarrow X$ for which $\bar{\nabla}^{t}=d y(\partial / \partial y)+\theta^{t}+\nabla_{(\cdot)}^{t}$, a two parameter family 
$\left\{D_{y}^{t}\right\},(t, y) \in T \times Y$, of Dirac operators on $C^{\infty}(X, S \otimes E)$ coupled to $\nabla_{y}^{t}$ and a one parameter family $\left\{H^{t}\right\}, t \in T$, of Dirac operators on $C^{\infty}(Z, \bar{S} \otimes \bar{E})$.

Recall (Proposition (3.6.3)) that $\xi(H) \bmod 1$ depends smoothly on $H$, and so the assignment $t \rightarrow \xi\left(H^{t}\right)$ defines a smooth map $T \mapsto \mathbf{R} / \mathbf{Z}$. With a little abuse of notation, let $(d \xi / d t) d t$ denote the pullback by this map of the unit normalized volume oneform on $\mathbf{R} / \mathbf{Z}$.

We will prove

Proposition (4.1). As one-forms on $T$,

$$
\begin{aligned}
\frac{d}{d t} \xi(H) d t= & {\left[\int_{Z} \hat{A}\left(\mathscr{R}_{g}\right) \operatorname{ch}\left(\mathscr{F}_{d t(\partial / \partial t)+\nabla}\right)\right]_{\text {one-form }} } \\
& +\frac{1}{2 \pi i} \sigma\left(D_{\infty}, \frac{d D_{\infty}}{d t} d t\right)-\frac{1}{2 \pi i} \sigma\left(D_{-\infty}, \frac{d D_{-\infty}}{d t} d t\right) .
\end{aligned}
$$

Here $\mathscr{F}_{d t(\partial / \partial t)+\bar{\nabla}}$ is the curvature of $d t(\partial / \partial t)+\bar{\nabla}$ viewed as a connection on the pullback of $\bar{E}$ to the bundle $T \times \bar{E}$ over $T \times Z$. Explicitly,

$$
\mathscr{F}_{d t(\partial / \partial t)+\bar{\nabla}}=\mathscr{F}_{\bar{\nabla}}+\frac{d \bar{\nabla}}{d t} d t \in \Omega^{2}(T \times Z, \operatorname{End}(T \times \bar{E})),
$$

where for each $t, \mathscr{F}_{\bar{\nabla}^{t}}$ is the curvature of $\bar{\nabla}^{t}$ as a connection on $\bar{E}$.

The first term on the right-hand side of (4.2) would give the complete expression for the derivative of $\xi(H)$ if $Z$ were compact without boundary. The last two terms give corrections due to the non-compactness.

Let $\mathscr{C}$ be a contour in $\mathbf{C}$ as in the definition of $\hat{\eta}^{\delta}(H)$. Define

$$
\begin{aligned}
& \hat{\mathscr{V}} \delta\left(H, \frac{d H}{d t}\right)(z)=-z \frac{d H}{d t}\left(H^{2}\right)^{-z-1 / 2}=z \int_{\mathscr{C}} \frac{d \lambda}{2 \pi i} \lambda^{-z-1 / 2} \frac{d H}{d t}\left(H^{2}-\lambda\right)^{-1}, \quad \operatorname{Re} z>0 \\
& \hat{\mathscr{S} \delta}\left(H, \frac{d H}{d t}\right)(z)=--\frac{1}{2} \Gamma \int_{C^{2}} \frac{d \lambda}{2 \pi i} \lambda^{-z-1 / 2}\left(\hat{\mathscr{S}}_{1}\left(H, \frac{d H}{d t}\right)(\lambda)\right. \\
&\left.+\left\{H, \hat{\mathscr{S}}_{2}\left(H, \frac{d H}{d t}\right)(\lambda)\right\}\right) \quad \operatorname{Re} z>0 \\
& \hat{\mathscr{S}}_{1}\left(H, \frac{d H}{d t}\right)(\lambda)=\left(H^{2}-\lambda\right)^{-1} \frac{d H}{d t} H\left(H^{2}-\lambda\right)^{-1} \\
& \hat{\mathscr{S}}_{2}\left(H, \frac{d H}{d t}\right)(\lambda)=-\left(H^{2}-\lambda\right)^{-1} \frac{d H}{d t}\left(H^{2}+\lambda\right)\left(H^{2}-\lambda\right)^{-2} \\
& \mathscr{V}_{\phi}^{\delta}\left(H, \frac{d H}{d t}\right)(z)=\operatorname{Tr} \phi \hat{\mathscr{V}}^{\delta}\left(H, \frac{d H}{d t}\right)(z), \quad \operatorname{Re} z>\operatorname{dim} Z \\
& \mathscr{S}_{\phi}^{\delta}\left(H, \frac{d H}{d t}\right)(z)=\operatorname{Tr} \phi \hat{\mathscr{S}}^{\delta}\left(H, \frac{d H}{d t}\right)(z), \quad \operatorname{Re} z>\operatorname{dim} Z
\end{aligned}
$$


As we will see below, $\mathscr{V}_{\phi}^{\delta}$ and $\mathscr{S}_{\phi}^{\delta}$ give respectively the "volume" and "surface" contributions to $d \xi / d t$.

By using psedudo-differential operator techniques and then Gilkey's Theorem as in the previous section, we see that for $\operatorname{Re} z>\frac{1}{2} \operatorname{dim} Z, \hat{\eta}^{\delta}(H, d H / d t)(z)$ and $\hat{\mathscr{V}}^{\delta}(H, d H / d t)(z)$ are bounded operators with continuous kernels, and these kernels extend to analytic functions of $z$ for $\operatorname{Re} z>-\frac{1}{2}$. In addition, because of the extra factor of $z$ in the definition of $\hat{\mathscr{V}}^{\delta}$, the pseudo-differential operator analysis, together with the results of Appendix $C$ shows that $\lim _{z \rightarrow 0} \operatorname{tr} \widehat{\mathscr{V}}^{\delta}(H, d H / d t)(z ; y, x, y, x)$ is given by a universal polynomial in the components of $\bar{g},(\operatorname{det} \bar{g})^{-1}$, and the covariant derivatives of $\mathscr{F}_{\bar{\nabla}}, \mathscr{R}_{\bar{g}}$, and $d H / d t$. We will give the well-known explicit formula for this quantity in Lemma (4.8) below.

Proposition (4.1) will follow by taking the limit $\phi \rightarrow 1$ and analytic continuation to $z=0$ of the following formula.

Lemma (4.6). For $\operatorname{Re} z$ sufficiently large,

$$
\frac{d}{d t} \eta_{\phi}^{\delta}(H)(z)=2 \mathscr{V}_{\phi}^{\delta}\left(H, \frac{d H}{d t}\right)(z)+2 \mathscr{S}_{d \phi / d y}^{\delta}\left(H, \frac{d H}{d t}\right)(z) .
$$

Proof. Formally

$$
\begin{aligned}
\frac{d}{d t} \eta_{\phi}^{\delta}(H)(z)= & -\operatorname{Tr} \int_{\mathscr{C}} \frac{d \lambda}{2 \pi i} \lambda^{-z-1 / 2} \phi \frac{d}{d t}\left(H\left(H^{2}-\lambda\right)^{-1}\right) \\
= & -\operatorname{Tr} \int_{\mathscr{C}} \frac{d \lambda}{2 \pi i} \lambda^{-z-1 / 2} \phi\left(\frac{d H}{d t}\left(H^{2}-\lambda\right)^{-1}\right. \\
& \left.-H\left(H^{2}-\lambda\right)^{-1} \frac{d H^{2}}{d t}\left(H^{2}-\lambda\right)^{-1}\right) \\
= & -\operatorname{Tr} \int_{\mathscr{C}} \frac{d \lambda}{2 \pi i} \lambda^{-z-1 / 2}\left(\phi \frac{d H}{d t}\left(H^{2}-\lambda\right)^{-1}\right. \\
& -H\left(H^{2}-\lambda\right)^{-1} \phi \frac{d H}{d t} H\left(H^{2}-\lambda\right)^{-1} \\
& \left.-H^{2}\left(H^{2}-\lambda\right)^{-1} \phi \frac{d H}{d t}\left(H^{2}-\lambda\right)^{-1}\right) \\
& -\operatorname{Tr} \int_{\mathscr{C}} \frac{d \lambda}{2 \pi i} \lambda^{-z-1 / 2}\left([H, \phi]\left(H^{2}-\lambda\right)^{-1} \frac{d H}{d t} H\left(H^{2}-\lambda\right)^{-1}\right. \\
& -H\left(H^{2}-\lambda\right)^{-1}\left[H^{2}, \phi\right]\left(H^{2}-\lambda\right)^{-1} \frac{d H}{d t} H\left(H^{2}-\lambda\right)^{-1} \\
& +\left[H^{2}, \phi\right]\left(H^{2}-\lambda\right)^{-1} \frac{d H}{d t}\left(H^{2}-\lambda\right)^{-1} \\
& \left.-H^{2}\left(H^{2}-\lambda\right)^{-1}\left[H^{2}, \phi\right]\left(H^{2}-\lambda\right)^{-1} \frac{d H}{d t}\left(H^{2}-\lambda\right)^{-1}\right)
\end{aligned}
$$




$$
\begin{aligned}
= & -\operatorname{Tr} \int_{\mathscr{C}} \frac{d \lambda}{2 \pi i} \lambda^{-z-1 / 2} \phi \frac{d H}{d t}\left(\left(H^{2}-\lambda\right)^{-1}-2\left(H^{2}-\lambda\right)^{-2} H^{2}\right) \\
- & \operatorname{Tr} \int_{\mathscr{C}} \frac{d \lambda}{2 \pi i} \lambda^{-z-1 / 2}[H, \phi]\left(\left(H^{2}-\lambda\right)^{-1} \frac{d H}{d t} H\left(H^{2}-\lambda\right)^{-1}\right. \\
& \left.-\left\{H,\left(H^{2}-\lambda\right)^{-1} \frac{d H}{d t}\left(H^{2}+\lambda\right)\left(H^{2}-\lambda\right)^{-2}\right\}\right) \\
= & 2 \operatorname{Tr} \phi \hat{\mathscr{V}}^{\delta}\left(H, \frac{d H}{d t}\right)(z)+2 \operatorname{Tr} \frac{d \phi}{d y} \hat{\mathscr{S}}^{\delta}\left(H, \frac{d H}{d t}\right)(z) .
\end{aligned}
$$

To obtain $(4.7 \mathrm{~d})$ we have cyclically permuted the order of operators under the trace and to obtain (4.7e) we have combined terms by integration by parts in $\lambda$.

The only subtlety in this formal calculation is the cyclic permutation of operators. This can be justified for each summand by integration by parts in $\lambda$ using the fact that for $N$ sufficiently large $\left(H^{2}-\lambda\right)^{-N} \phi$ is trace class. (See $[8,12]$ for details).

We next compute $\mathscr{V}_{\phi}^{\delta}$ and $\mathscr{S}_{\phi}^{\delta}$ in two important cases.

\section{Lemma (4.8).}

1. $\operatorname{tr} \hat{\mathscr{V}}^{\delta}(H,(d H / d t) d t)(0 ; y, x, y, x) d y|d x|$ is the differential form on $T \times Y \times X$ given by the term of degree $(1,1, \operatorname{dim} X)$ of $\hat{A}\left(\mathscr{R}_{g}\right) \operatorname{ch}\left(\mathscr{F}_{d t(\hat{\partial} / \partial t)+\bar{\nabla})}\right.$.

2. Suppose that for all $t, H^{t}$ is invariant under translations of $Y$. Then for $\phi$ of compact support with $\int_{R} \phi=1$,

$$
\begin{aligned}
& \mathscr{S}_{\phi}^{\delta}\left(H, \frac{d H}{d t}\right)(z)=\frac{1}{2 \pi i} \frac{\Gamma\left(\frac{1}{2}\right)}{\Gamma\left(z+\frac{1}{2}\right)} \Gamma(z+1) \sigma\left(D_{ \pm \infty}, \frac{d D_{ \pm \infty}}{d t}\right)(z), \\
& \hat{\mathscr{V}}^{\delta}\left(H, \frac{d H}{d t}\right)(z)=0 .
\end{aligned}
$$

As in Lemma (3.13), the hypothesis of statement (4.8.2) means that for each $t, \theta^{t}=0$ and $D_{y}^{t}=D_{ \pm \infty}^{t}$ for all $y$. Note that for arbitrary $H^{t}$, this hypothesis is satisfied by the operators $H_{ \pm \infty}^{t}$ defined in (3.12).

Since $\operatorname{tr} \hat{\mathscr{V}}^{\delta}(0 ; y, x, y, x) d y|d x|$ is given by a local expression (see Appendix C), (4.8.1) follows directly from the corresponding well known result for compact odd dimensional manifolds (see, e.g. [4]). (4.8.2) follows from an easy calculation using the expression (3.15) for $\left(\left(H^{t}\right)^{2}-\lambda\right)^{-1}$.

We now want to take the limits of $\mathscr{V}_{\phi}^{\delta}(H, d H / d t)(z)$ and $\mathscr{S}_{d \phi / d y}^{\delta}(H, d H / d t)$ as $\phi$ approaches the constant function 1 . As in the definition of $\eta$ we can obtain the necessary estimates by comparing these quantities for arbitrary $H$ with the corresponding quantities computed for the operators $H_{ \pm \infty}$ defined in (3.12). Define

$$
\begin{aligned}
\Delta_{ \pm} \hat{\mathscr{V}}^{\delta}(z) & =\hat{\mathscr{V}}^{\delta}\left(H, \frac{d H}{d t}\right)(z)-\hat{\mathscr{V}}^{\delta}\left(H_{ \pm \infty}, \frac{d H_{ \pm \infty}}{d t}\right)(z), \\
\Delta_{ \pm} \hat{\mathscr{S}}_{1,2}^{\delta}(\lambda) & =\hat{\mathscr{S}}_{1,2}^{\delta}\left(H, \frac{d H}{d t}\right)(\lambda)-\hat{\mathscr{S}}_{1,2}^{\delta}\left(H_{ \pm \infty}, \frac{d H_{ \pm \infty}}{d t}\right)(\lambda) .
\end{aligned}
$$


For $F \subset \mathbf{C}$, let $\rho_{ \pm}(F)$ be the square root of the distance from $F$ to the spectrum of $D_{ \pm \infty}$.

\section{Lemma (4.11).}

1. There exists a constant $c$ such that for $y>1$ and $\lambda \in \mathscr{C}, \Delta_{+} \hat{\mathscr{S}}_{1}(\lambda ; y, x, y, x)$ is continuous in $y, x, \lambda$, and $\left|\Delta_{+} \hat{\mathscr{S}}_{1}(\lambda ; y, x, y, x)\right|<c \exp -\rho_{+}(\lambda)|y|$. An analogus statement holds for $\Delta_{+} \hat{\mathscr{S}}_{2}$.

2. For $y>1, \Delta_{+} \hat{\mathscr{S}}^{\delta}(z ; y, x, y, x)$ extends to an entire function of $z$.

3. For any compact set $K \subset \mathbf{C}$ there exists a constant $c$ such that for $z \in K$ and $y>1$,

$$
\left|\Delta_{+} \hat{\mathscr{S}}^{\delta}(z ; y, x, y, x)\right|<c \exp -\rho_{+}(\mathscr{C})|y| .
$$

These statements hold under the simultaneous replacement of $y>1$ by $y<$ $-1, \Delta_{+}$by $\Delta_{-}$, and $\rho_{+}$by $\rho_{-}$. Analogous statements hold with $\hat{\mathscr{S}}^{\delta}$ replaced by $\hat{\mathscr{V}}^{\delta}$.

Proof. We will prove (4.11.1). (4.11.2) and (4.11.3) then follow easily by integrating over $\lambda$ and using the uniformity of the bound in (4.11.1).

As bounded operators between the appropriate Sobolev spaces,

$$
\begin{aligned}
\Delta_{+} \hat{\mathscr{S}}_{1}\left(H, \frac{d H}{d t}\right)(\lambda)= & \left(H^{2}-\lambda\right)^{-1} \frac{d H}{d t} H\left(H^{2}-\lambda\right)^{-1} \\
& -\left(H_{\infty}^{2}-\lambda\right)^{-1} \frac{d H_{\infty}}{d t} H_{\infty}\left(H_{\infty}^{2}-\lambda\right)^{-1} \\
= & \left(\left(H^{2}-\lambda\right)^{-1}-\left(H_{\infty}^{2}-\lambda\right)^{-1}\right) \frac{d H}{d t} H\left(H^{2}-\lambda\right)^{-1} \\
& +\left(H_{\infty}^{2}-\lambda\right)^{-1}\left(\frac{d H}{d t} H-\frac{d H_{\infty}}{d t} H_{\infty}\right)\left(H^{2}-\lambda\right)^{-1} \\
& +\left(H_{\infty}^{2}-\lambda\right)^{-1} \frac{d H_{\infty}}{d t} H_{\infty}\left(\left(H^{2}-\lambda\right)^{-1}-\left(H_{\infty}^{2}-\lambda\right)^{-1}\right),
\end{aligned}
$$

and also

$$
\begin{aligned}
\left(H^{2}-\lambda\right)^{-1}-\left(H_{\infty}^{2}-\lambda\right)^{-1} & =\left(H_{\infty}^{2}-\lambda\right)^{-1}\left(H_{\infty}^{2}-H^{2}\right)\left(H^{2}-\lambda\right)^{-1}, \\
H_{\infty}^{2}-H^{2} & =H_{\infty}\left(H_{\infty}-H\right)+\left(H_{\infty}-H\right) H, \\
\frac{d H}{d t} H-\frac{d H_{\infty}}{d t} H_{\infty} & =\frac{d H}{d t}\left(H-H_{\infty}\right)+\left(\frac{d H}{d t}-\frac{d H_{\infty}}{d t}\right) H_{\infty} .
\end{aligned}
$$

Combining these expressions, we can write $\Delta_{+} \hat{\mathscr{S}}_{1}(H, d H / d t)(\lambda)$ as the sum of terms of the form $\left(H_{\infty}^{2}-\lambda\right)^{-1} \mathscr{L}\left(H-H_{\infty}\right) \mathscr{P}, \mathscr{P}\left(H-H_{\infty}\right) \mathscr{L}\left(H_{\infty}^{2}-\lambda\right)^{-1},\left(H_{\infty}^{2}-\lambda\right)^{-1}$ $\mathscr{L}\left(d H / d t-d H_{\infty} / d t\right) \mathscr{P}$, and $\mathscr{P}\left(d H / d t-d H_{\infty} / d t\right) \mathscr{L}\left(H_{\infty}^{2}-\lambda\right)^{-1}$, where $\mathscr{L}$ is either $H, H_{\infty}, d H / d t$, or $d H_{\infty} / d t$, and $\mathscr{P}$ is a pseudo-differential operator of negative order formed from the composition of $\left(H^{2}-\lambda\right)^{-1},\left(H_{\infty}^{2}-\lambda\right)^{-1}, H, H_{\infty}, d H / d t$, and $d H_{\infty} / d t$. We will discuss the terms of the first form; the terms of the other forms can be treated similarly. Observe that for $y>1$,

$$
\begin{aligned}
& {\left[\left(H_{\infty}^{2}-\lambda\right)^{-1} \mathscr{L}\left(H-H_{\infty}\right) \mathscr{P}\right](y, x ; y, x)} \\
& \quad=\int_{X} d x^{\prime} \int_{y^{\prime}<1} d y^{\prime}\left(H_{\infty}^{2}-\lambda\right)^{-1}\left(y, x ; y^{\prime}, x^{\prime}\right)\left(\mathscr{L}\left(H-H_{\infty}\right) \mathscr{P}\right)\left(y^{\prime}, x^{\prime} ; y, x\right) .
\end{aligned}
$$


The $y^{\prime}$ integration can be restricted to $y^{\prime}<1$ since $H-H_{\infty}$ is supported on $y^{\prime}<1$ and $\mathscr{L}$ is a local operator. The kernels for $\mathscr{P}$ and $\left(H_{\infty}^{2}-\lambda\right)^{-1}$ are smooth off the diagonal, so that for $y>1$ there are no singularities in the integrands of (4.16). The kernel $\mathscr{P}\left(y, x ; y^{\prime}, x^{\prime}\right)$ is bounded uniformly for $\lambda \in \mathscr{C}$ and $\left|y-y^{\prime}\right|>\varepsilon>0$. Moreover, by Proposition (A.6), the kernel $\left(H_{\infty}^{2}-\lambda\right)^{-1}\left(y, x ; y^{\prime}, x^{\prime}\right)$ decays exponentially as $e^{-\rho+(\lambda)|y-y|}$ for $\lambda \in \mathscr{C}$. Inserting these estimates into (4.16) and performing the $y^{\prime}$ and $x^{\prime}$ integrals we obtain

$$
\left|\left(H_{\infty}^{2}-\lambda\right)^{-1} \mathscr{L}\left(H-H_{\infty}\right) \mathscr{P}(y, x ; y, x)\right|<\mathrm{const} e^{-\rho+(\lambda)|y|}
$$

for $y>1$. This completes the proof of (4.11.1) and of the Proposition.

Finally, we give the

Proof of Proposition (4.1). The idea is take the limit $\phi \rightarrow 1$ and the analytic continuation to $z=0$ of the formula of Lemma (4.6). These limits exist because of the analyticity properties and decay estimates of Lemmas (2.8), (3.6), (3.7), (3.16) and (4.11). In the limit the terms on the right-hand side of (4.6) can be evaluated using Lemma (4.8). We now make this procedure precise.

Fix $\delta>0$ suitable for the definition of $\eta^{\delta}(H)(z)$ as in Sect. 3. By Propositions (2.8), (3.6), (3.7), and (4.11), the functions $\sigma\left(D_{ \pm \infty}^{t}, d D_{ \pm \infty}^{t} / d t\right), \quad \eta^{\delta}\left(H^{t}\right)(z), \quad \eta_{\phi}^{\delta}\left(H^{t}\right)(z)$, $\mathscr{V}_{\phi}^{\delta}\left(H^{t}, d H / d t\right)(z), \mathscr{S}_{\phi}^{\delta}\left(H^{t}, d H / d t\right)(z)$, defined originally for $t \in T$ and $\operatorname{Re} z$ large, extend to functions on $T \times\left\{\operatorname{Re} z>-\frac{1}{2}\right\}$ which for each $t$ are analytic in $z$. For notational simplicity, we denote these extended functions by $\sigma_{ \pm}(t, z), \eta(t, z), \eta_{\phi}(t, z), \mathscr{V}_{\phi}(t, z)$, $\mathscr{S}_{\phi}(t, z)$ respectively. Also denote by $c(t)$ the coefficient of $d t$ in the one form piece of $\int_{Z} \hat{A}\left(\mathscr{R}_{g}\right) \operatorname{ch}\left(\mathscr{F}_{d t(\partial / \partial t)+\bar{\nabla})}\right.$.

It suffices to show that

$$
\frac{1}{2} \frac{d}{d t}(\eta(t, 0))=c(t)+\frac{1}{2 \pi i} \sigma_{+}(t, 0)-\frac{1}{2 \pi i} \sigma_{-}(t, 0) .
$$

Let $\phi \in C_{0}^{\infty}(\mathbf{R})$ with $\phi(y)=1$ for $|y|<1$. Then for $\operatorname{Re}>-\frac{1}{2}$,

$$
\begin{gathered}
\eta_{\phi}(t, z)-\int_{|y|<1} d y \int_{X}|d x| \operatorname{tr} \hat{\eta}^{\delta}(t, z ; y, x, y, x) \\
=\int_{y<-1} d y \int_{X}|d x| \phi(y) \operatorname{tr} \Delta_{-} \hat{\eta}^{\delta}(t, z ; y, x, y, x) \\
\quad+\int_{y>1} d y \int_{X}|d x| \phi(y) \operatorname{tr} \Delta_{+} \hat{\eta}^{\delta}(t, z ; y, x, y, x), \\
\mathscr{S}_{d \phi / d y}(t, z)-\frac{1}{2 \pi i} \frac{\Gamma\left(\frac{1}{2}\right)}{\Gamma\left(z+\frac{1}{2}\right)} \Gamma(z+1)\left(\sigma_{+}(t, z)-\sigma_{-}(t, z)\right) \\
=\int_{y<-1} d y \int_{X}|d x| \frac{d \phi}{d y}(y) \operatorname{tr} \Delta_{-} \hat{\mathscr{S}}^{\delta}(t, z ; y, x, y, x) \\
\quad+\int_{y>1} d y \int_{X}|d x| \frac{d \phi}{d y}(y) \operatorname{tr} \Delta_{+} \hat{\mathscr{S}}^{\delta}(t, z ; y, x, y, x), \\
\mathscr{V}_{\phi}(t, z)-c(t)=\int_{|y|<1} d y \int_{X}|d x| \operatorname{tr}\left(\hat{\mathscr{V}}^{\delta}(t, z ; y, x, y, x)-\hat{\mathscr{V}}^{\delta}(t, 0 ; y, x, y, x)\right)
\end{gathered}
$$




$$
\begin{aligned}
& +\int_{y<-1} d y \int_{X}|d x| \phi(y) \operatorname{tr} \Delta_{-} \hat{\mathscr{V}}^{\delta}(t, z ; y, x, y, x) \\
& +\int_{y>1} d y \int_{X}|d x| \phi(y) \operatorname{tr} \Delta_{+} \hat{\mathscr{V}}^{\delta}(t, z ; y, x, y, x) .
\end{aligned}
$$

In fact, for $\operatorname{Re} z$ large, these equations follow from the definitions and Lemma (4.8). They continue to hold by analytic continuation for $\operatorname{Re} z>-\frac{1}{2}$ by Lemmas (3.16) and (4.11).

From (4.19), (4.20), (4.21), and the decay estimates of Lemmas (3.16) and (4.11), for the operator kernels, we deduce that for any compact set $\mathscr{K}$ in $T \times\left\{\operatorname{Re} z>-\frac{1}{2}\right\}$, the limits of $\eta_{\phi}, \mathscr{S}_{d \phi / d y}, \mathscr{V}_{\phi}$, as $\phi$ increases pointwise to the constant function 1 , exist uniformly for $(t, z) \in \mathscr{K}$. Moreover, by (3.7) and its analog for $\mathscr{S}_{d \phi / d y}$ and $\mathscr{V}_{\phi}$, the functions $\eta_{\phi}, \mathscr{S}_{d \phi / d y}, \mathscr{V}_{\phi}$, are uniformly continuous on $\mathscr{K}$.

In particular, for $(t, z) \in T \times\left\{\operatorname{Re} z>-\frac{1}{2}\right\}$,

$$
\begin{aligned}
\lim _{\phi \rightarrow 1}\left(\eta_{\phi}(t, z)\right) & =\left(\lim _{\phi \rightarrow 1} \eta_{\phi}\right)(t, z)=\eta(t, z), \\
\lim _{\phi \rightarrow 1}\left(\mathscr{S}_{d \phi / d y}(t, z)\right) & =\frac{1}{2 \pi i} \frac{\Gamma\left(\frac{1}{2}\right)}{\Gamma\left(z+\frac{1}{2}\right)} \Gamma(z+1)\left(\sigma_{+}(t, z)-\sigma_{-}(t, z)\right), \\
\lim _{\phi \rightarrow 1}\left(\mathscr{V}_{\phi}(t, 0)\right) & =c(t) .
\end{aligned}
$$

In (4.24) we have used $\Delta_{ \pm} \hat{\mathscr{V}}^{\delta}(t, 0 ; y, x, y, x)=0$ for $|y|>1$ as follows from Lemma (4.8).

Now, for $(t, z) \in T \times\left\{\operatorname{Re} z>-\frac{1}{2}\right\}$,

$$
\eta_{\phi}(t, z)=\eta_{\phi}(0, z)+2 \int_{0}^{t} d s\left(\mathscr{S}_{\phi}(s, z)+\mathscr{V}_{\phi}(s, z)\right)
$$

In fact, (4.25) holds for $\operatorname{Re} z$ large by Lemma (4.6). On the other hand, by the uniform continuity on compact sets in $(s, z)$ of the integrands, the Riemann sums defining the integrals on the right-hand side of (4.25) converge uniformly in $z$ on compacts sets for $\operatorname{Re} z>-\frac{1}{2}$. Thus both sides of (4.25) define analytic functions of $z$ for $\operatorname{Re} z>-\frac{1}{2}$, and so (4.25) holds for $\operatorname{Re} z>-\frac{1}{2}$.

Taking the limit as $\phi \rightarrow 1$ of (4.25), and using uniform convergence to interchange the order of limit and integration, we deduce that for $\operatorname{Re} z>-\frac{1}{2}$,

$$
\lim _{\phi \rightarrow 1}\left(\eta_{\phi}(t, z)\right)=\lim _{\phi \rightarrow 1}\left(\eta_{\phi}(0, z)\right)+2 \int_{0}^{t} d s\left[\lim _{\phi \rightarrow 1}\left(\mathscr{S}_{\phi}(s, z)\right)+\lim _{\phi \rightarrow 1}\left(\mathscr{V}_{\phi}(s, z)\right)\right]
$$

In particular, for $z=0,(4.26)$ together with (4.22)-(4.24) implies

$$
\eta(t, 0)=\lim _{\phi \rightarrow 1}\left(\eta_{\phi}(t, 0)\right)=\lim _{\phi \rightarrow 1}\left(\eta_{\phi}(0,0)\right)+2 \int_{0}^{t} d s\left[c(s)+\frac{1}{2 \pi i} \sigma_{+}(s, 0)+\frac{1}{2 \pi i} \sigma_{-}(s, 0)\right] .
$$

Equation (4.18) follows by differentiating (4.27) with respect to $t$, and the proof is complete. 


\section{Parallel Transport for $\nabla^{\mathscr{L}}$}

In this section we will prove our parallel transport formula, Theorem (1.10), by integrating the formula of Proposition (4.1) over $T$. We continue with the notation and assumptions of the previous section. Thus $\left\{\bar{\nabla}^{t}\right\}$ denotes a smooth family parametrized by $T$ of connections on $\bar{E} \rightarrow Z=\mathbf{R} \times X$ with $\bar{\nabla}^{t}=d y(\partial / \partial y)+\theta_{(\cdot)}^{t}+\nabla_{(\cdot)}^{t}$. We assume that for all $t, \bar{\nabla}^{t}$ satisfies conditions 1 and 2 , and that for $|t|$ large, $\bar{\nabla}^{t}$ is independent of $t$.

We first integrate the volume piece of (4.1). Let

$$
\overline{\bar{\nabla}}=d t \frac{\partial}{\partial t}+\bar{\nabla}=d t \frac{\partial}{\partial t}+d y \frac{\partial}{\partial y}+\theta+\nabla,
$$

which we view as a connection on the pullback $T \times \bar{E}$ of $\bar{E}$ to $T \times Z$. Define

$$
I(\overline{\bar{\nabla}})=\int_{T \times Z} \hat{A}\left(\mathscr{R}_{g}\right) \operatorname{ch}\left(\mathscr{F}_{\bar{\nabla}}\right) .
$$

\section{Lemma (5.3).}

1. Suppose $\theta^{t}=0$ and $\nabla_{y}^{t}=\nabla_{t}^{y}$ for all $(t, y)$. Then $I(\overline{\bar{\nabla}})=0$.

2. Suppose $\nabla_{y}^{t}=\nabla$ is independent of $t$. Then

$$
I(\overline{\bar{\nabla}})=-\frac{1}{2 \pi i} \int_{Z} \hat{A}\left(\mathscr{R}_{g}\right) \operatorname{tr}\left(\theta^{(t=\infty)}-\theta^{(t=-\infty)}\right) \exp i \mathscr{F}_{\nabla} / 2 \pi .
$$

Proof. To prove (5.3.1), let $\mu: T \times Y \times X \mapsto T \times Y \times X$ be the map which interchanges the first two factors, $\mu(t, y, x)=(y, t, x)$. Then the assumption of (5.3.1) implies that $\mathscr{F}_{\bar{\nabla}}$ is invariant under $\mu^{*}$, and so the integrand in (5.2) is also invariant under $\mu^{*}$. However since $\mu$ is orientation reversing, $\int \alpha=-\int \mu^{*} \alpha$ for any compactly supported differential form $\alpha$ on $T \times Y \times X$. Thus, $I(\overline{\bar{\nabla}})=0$.

Under the hypothesis of (5.3.2), $\mathscr{F}_{\bar{\nabla}}=\mathscr{F}_{\nabla}+d t(d \theta / d t)$ and (5.3.2) follows easily.

We can now give the

Proof of Theorem (1.10). It suffices to prove the equality of the phases

$$
\xi(H)=\frac{1}{2 \pi i} \int_{Y} \sigma(D, \nabla D) \bmod 1 .
$$

Write $\bar{\nabla}=d y(\partial / \partial y)+\theta+\nabla_{(\cdot)}$. We will prove (5.4) by integrating the equation of Proposition (4.1) along interpolating families of connections parametrized by $T$ from $d y(\partial / \partial y)+\nabla_{-\infty}$ to $d y(\partial / \partial y)+\nabla_{(\cdot)}$ and from $d y(\partial / \partial y)+\nabla_{(\cdot)}$ to $\bar{\nabla}$.

First, choose an interpolating family of connections $\left\{\bar{\nabla}^{t}\right\}$ on $\bar{E}$ as in Lemma (5.3.1) with $\bar{\nabla}^{(t<-3)}=d y(\partial / \partial y)+\nabla_{-\infty}$ and $\bar{\nabla}^{(t>3)}=d y(\partial / \partial y)+\nabla_{(\cdot)}$. (This can be done as follows. Let $\alpha$ be a nondecreasing smooth function on $Y$ with $\alpha(y)=-2$ for $y<-3, \alpha(y)=2$ for $y>3$, and $\alpha(y)=y$ for $-1<y<1$. Note that $\nabla_{y}=\nabla_{\alpha(y)}$ for all $y$ since $\nabla_{y}$ is independent of $y$ for $|y|>1$. Let $\beta$ be a smooth function on $T \times Y$ with $\beta(t, y)=-2$ for $t<-3, \beta(t, y)=\alpha(y)$ for $t>3$, and $\beta(t, y)=\beta(y, t)$ for all $t, y$. Then set $\left.\nabla_{y}^{t}=\nabla_{\beta(t, y)}\right)$ 
Now integrate Eq. (4.1) over T:

$$
\begin{aligned}
& \xi\left(H^{(t=\infty)}\right)-\xi\left(H^{(t=-\infty)}\right) \\
& \quad=I(\overline{\bar{\nabla}})+\frac{1}{2 \pi i} \int_{T} \sigma\left(D_{\infty}^{(\cdot)}, \frac{d D_{\infty}^{(\cdot)}}{d t} d t\right)-\frac{1}{2 \pi i} \int_{T} \sigma\left(D_{-\infty}^{(\cdot)}, \frac{d D_{-\infty}^{(\cdot)}}{d t} d t\right) \bmod 1 .
\end{aligned}
$$

For this family of connections $\left\{\bar{\nabla}^{t}\right\}, \xi\left(H^{(t=-\infty)}\right)=0$ by Lemma $(3.13), I(\bar{\nabla})=0$ by Lemma (5.3.1), and the last term on the right-hand side of (5.5) also vanishes since $D_{-\infty}^{(\cdot)}$ is independent of $t$. Finally, because of the symmetry $\nabla_{y}^{t}=\nabla_{t}^{y}$ we can interchange the roles of $t$ and $y$ in the second term on the right-hand side. We obtain

$$
\xi\left(H_{d y(\partial / \partial y)+\nabla_{(\cdot)}}\right)=\frac{1}{2 \pi i} \int_{Y} \sigma\left(D, \frac{d D}{d y} d y\right) \bmod 1 .
$$

Next, choose an interpolating family of connections $\left\{\bar{\nabla}^{t}\right\}$ on $\bar{E}$ as in Lemma (5.3.2) with $\bar{\nabla}^{(t<-1)}=d y(\partial / \partial y)+\nabla_{(\cdot)}$ and $\bar{\nabla}^{(t>1)}=\bar{\nabla}$. For this family both $D_{\infty}^{t}$ and $D_{-\infty}^{t}$ are independent of $t$ and so the surface terms $\sigma$ in (4.1) vanish. In addition, by combining Lemma (5.3.2) with Proposition (2.8.3), we see that

$$
I(\bar{\nabla})=-\frac{1}{2 \pi i} \int_{Y} \int_{X} \hat{A}\left(\mathscr{R}_{g}\right) \operatorname{tr} \theta \exp i \mathscr{F} \nabla / 2 \pi=\frac{1}{2 \pi i} \int_{Y} \sigma(D,[\theta, D]) .
$$

Thus, integrating (4.1) for this family we obtain

$$
\xi\left(H_{\bar{\nabla}}\right)-\xi\left(H_{d y(\partial / \partial y)+\nabla_{(\cdot)}}\right)=\frac{1}{2 \pi i} \int_{Y} \sigma(D,[\theta, D]) \bmod 1 .
$$

Since $\nabla D=(d D / d y) d y+[\theta, D],(5.4)$ follows by adding (5.6) and (5.8).

\section{The Curvature of $\nabla^{\mathscr{L}}$}

In this section we prove the curvature formula, Theorem (1.5), as an additional application of Proposition (4.1).

First observe that if we take the parameter space $T$ to be a more general smooth manifold than $T=\mathbf{R}$, then Proposition (4.1) generalizes to the statement that as one forms on $T$,

$$
\xi^{*} d u=\left[\int_{Z} \hat{A}\left(\mathscr{R}_{g}\right) \operatorname{ch}\left(\mathscr{F}_{d_{T}+\bar{\nabla}}\right)\right]_{\text {one-form }}+\frac{1}{2 \pi i} \sigma\left(D_{\infty}, d_{T} D_{\infty}\right)-\frac{1}{2 \pi i} \sigma\left(D_{-\infty}, d_{T} D_{-\infty}\right) .
$$

Here we view $t \mapsto \xi\left(H^{t}\right)$ as a smooth function $\xi: T \mapsto \mathbf{R} / \mathbf{Z}, d u$ is the standard one-form on $\mathbf{R} / \mathbf{Z}, \xi^{*} d u$ is its pull-back to a one form on $T$, and $d_{T}$ is the exterior derivative of forms on $T$. From this we obtain the following

Corollary (6.2). As two-forms on $T$,

$$
d_{T}\left(\sigma\left(D_{\infty}, d_{T} D_{\infty}\right)\right)=2 \pi i\left[\int_{X} \hat{A}\left(\mathscr{R}_{g}\right) \operatorname{ch}\left(\mathscr{F}_{d_{T}+\nabla_{\infty}}\right)\right]_{\{\text {two-form }\}} .
$$


Note that except for the subscript on $D_{\infty}$, the space $Y=\mathbf{R}$ does not enter into the above formula.

Proof. Choose a family of connections $\left\{\bar{\nabla}^{t}\right\}, t \in T$, on $\bar{E}$ as above, satisfying the hypothesis of Theorem (1.10) such that $\nabla_{-\infty}^{t}$ is independent of $t$. For this family, the exterior derivative of $(6.1)$ is

$$
0=2 \pi i\left[d_{T} \int_{Z} \hat{A}\left(\mathscr{R}_{g}\right) \operatorname{ch}\left(\mathscr{F}_{d_{T}+\bar{\nabla}}\right)\right]_{\text {two-form }}+d_{T}\left(\sigma\left(D_{\infty}, d_{T} D_{\infty}\right)\right)
$$

It thus remains to simplify the first term on the right-hand side of (6.3).

Let $\Phi=\hat{A}\left(\mathscr{R}_{g}\right) \operatorname{ch}\left(\mathscr{F}_{d_{T}+\bar{\nabla}}\right) \in \Omega *(T \times Y \times X)$. Then by the usual Chern-Weil algebra, $\left(d_{T}+d_{Y}+d_{X}\right) \Phi=0$, and so as differential forms on $T$,

$$
d_{T}\left[\int_{Y \times X} \Phi\right]=\int_{Y \times X} d_{T} \Phi=-\int_{Y \times X}\left(d_{Y}+d_{X}\right) \Phi=-\int_{\partial(Y \times X)} \Phi=-\left.\int_{X} \Phi\right|_{y=-\infty} ^{y=\infty} .
$$

The piece of $\Phi$ of degree 0 in $Y$ is $\widehat{A}\left(\mathscr{R}_{g}\right) \operatorname{ch}\left(\mathscr{F}_{d_{T}+\nabla}\right)$, and $\mathscr{F}_{d_{T}+\nabla}$ vanishes at $y=-\infty$ since $\nabla_{-\infty}^{t}$ is independent of $t$. The corollary thus follows by combining (6.3) and (6.4).

Now in (6.1) and the proof of (6.2) we are assuming that $Y$ is the real line $Y=\mathbf{R}$. For the curvature formula (1.5) we consider instead an arbitrary parameter manifold $Y$ as in Sect. 2.

Proof of Theorem (1.5). From Proposition (2.8.2) of Sect. 2, $d_{Y} \omega=d_{Y}(\sigma(D, \nabla D))$, so we must prove that as two forms on $Y$,

$$
d_{Y}(\sigma(D, \nabla D))=2 \pi i\left[\int_{X} \hat{A}\left(\mathscr{R}_{g}\right) \operatorname{ch}\left(\mathscr{F}_{\bar{\nabla}}\right)\right]_{\{\text {two-form }\}} \cdot
$$

Here as usual $\nabla D=d_{Y} D+[\theta, D] \quad$ and $\quad \bar{\nabla}=d_{Y}+\theta+\nabla_{(\cdot)} \quad$ with $\theta \in \Omega_{1}(Y) \otimes C^{\infty}(X$, Ene $E)$.

From (6.2) with $T$ replaced by $Y$, we deduce

$$
d_{Y}\left(\sigma\left(D, d_{Y} D\right)\right)=2 \pi i\left[\int_{X} \hat{A}\left(\mathscr{R}_{g}\right) \operatorname{ch}\left(\mathscr{F}_{d_{Y}+\nabla}\right)\right]_{\{\text {two-form }\}} \cdot
$$

From Proposition (2.8.3) of Sect. 2,

$$
d_{Y}(\sigma(D,[\theta, D]))=-d_{Y} \int_{X} \hat{A}\left(\mathscr{R}_{g}\right) \operatorname{tr} \theta \exp i \mathscr{F}_{\nabla} / 2 \pi .
$$

Finally, a standard calculation using $\operatorname{ch}(\mathscr{F})=\operatorname{tr} \exp i \mathscr{F} / 2 \pi$ shows that as two-forms on $Y$,

$$
\begin{aligned}
& 2 \pi i {\left[\int_{X} \hat{A}\left(\mathscr{R}_{g}\right) \operatorname{ch}\left(\mathscr{F}_{d_{Y}+\theta+\nabla}\right)\right]_{\{\text {two-form }\}} } \\
& \quad=2 \pi i\left[\int_{X} \hat{A}\left(\mathscr{R}_{g}\right) \operatorname{ch}\left(\mathscr{F}_{d_{Y}+\nabla}\right)\right]_{\{\text {two-form }\}}-d_{Y} \int_{X} \hat{A}\left(\mathscr{R}_{g}\right) \operatorname{tr} \theta \exp \mathscr{F}_{\nabla} .
\end{aligned}
$$

Equation (6.5) follows by combining (6.6), (6.7), and (6.8).

\section{A. The Spectra and Resolvents of $\partial^{\dagger} \partial, D^{2}$, and $H^{2}$}

In this Appendix we discuss the spectra and resolvents of the extensions to 
unbounded operators on $L_{0}^{2}$ of the operators $\partial^{\dagger} \partial, D^{2}$ and $H^{2}$. For our notational conventions, see Appendix D.

\section{Proposition (A.1).}

1. $D_{0,0}^{2}$ is self-adjoint with domain $L_{2}^{2}(X, S \otimes E) \subset L_{0}^{2}(X, S \otimes E)$.

2. The spectrum of $D_{0,0}^{2}$ consists of isolated points in $[0, \infty)$ corresponding to finite dimensional spaces of smooth eigenfunctions.

We omit the proof. Similar statements hold for $\partial \partial^{\dagger}$.

The properties of $H$ are a little more subtle since $\mathbf{R} \times X$ is not compact.

Proposition (A.2). Suppose that $H$ satisfies conditions 1 and 2 of Sect. 1. Then 1. $H_{0,0}$ is self-adjoint with domain $L_{2}^{2}(\mathbf{R} \times X, \bar{S} \otimes \bar{E})$.

2. Let $\lambda_{0}$ be a lower bound for the spectra of $\left(D_{\infty}\right)_{0,0}^{2}$ and $\left(D_{-\infty}\right)_{0,0}^{2}$. Then the essential spectrum of $H_{0,0}^{2}$ is contained in $\left[\lambda_{0}, \infty\right)$.

By the essential spectrum we mean those points of the spectrum which are not isolated eigenvalues of finite multiplicity. (See [11].) As we will see in the proof, this Proposition continues to hold if in place of condition 1 of Sect. 1 we only assume that the operators $D_{ \pm \infty}$ on the ends of the cylinder $\mathbf{R} \times X$ are invertible.

Proof. (A.2.1) is standard. For (A.2.2), write $H^{2}=h^{2}+K$ as an operator on $C_{0}^{\infty}(\mathbf{R} \times X, \bar{S} \otimes \bar{E})$, where

$$
h^{2}=-\frac{\partial^{2}}{\partial y^{2}}+D^{2}+\phi^{2}, \quad K=\left\{i \Gamma\left(\frac{\partial}{\partial y}+\theta\left(\frac{\partial}{\partial y}\right)\right), D\right\}-\phi^{2},
$$

with $\phi=\phi(y)$ any smooth nonnegative real-valued function on $Y$ of compact support which is identically $\lambda_{0}$ if $|y|<1$. Since the coefficients of $K$ are compactly supported on $\mathbf{R} \times X, K$ is $h^{2}$ compact by the Rellich lemma. Hence the closures $h_{0,0}^{2}$ and $H_{0,0}^{2}$ have the same domain, $H_{0,0}^{2}=h_{0,0}^{2}+K$, and $K$ is $h_{0,0}^{2}$-compact ([11], p. 194). Since $h_{0,0}^{2}$ is self-adjoint, it follows from a theorem of Weyl on the stability of the essential spectrum $\left([14]\right.$, p. 113) that $H_{0,0}^{2}$ and $h_{0,0}^{2}$ have the same essential spectrum.

It thus suffices to prove that the spectrum of $h_{0,0}^{2}$ is bounded from below by $\lambda_{0}$. Since $h_{0,0}^{2}$ is self-adjoint, this is equivalent to proving that $h_{0,0}^{2} \geqq \lambda_{0}$ as operators on $L_{0}^{2}(\mathbf{R} \times X, \bar{S} \otimes \bar{E})([11]$, p. 278). This follows from the computation

$$
\begin{aligned}
\left\langle\psi, h_{0,0}^{2} \psi\right\rangle \geqq & \int_{y<-1} d y\left\langle\psi(y, \cdot), D_{-\infty}^{2} \psi(y, \cdot)\right\rangle_{X}+\int_{y>1} d y\left\langle\psi(y, \cdot), D_{\infty}^{2} \psi(y, \cdot)\right\rangle_{X} \\
& +\int_{|y| \leqq 1} d y \phi^{2}(t)\langle\psi(y, \cdot), \psi(y, \cdot)\rangle_{X} \\
\geqq & \lambda_{0} \int d y\langle\psi(y, \cdot), \psi(y, \cdot)\rangle x=\lambda_{0}\langle f, f\rangle,
\end{aligned}
$$

where $\psi \in C_{0}^{\infty}(Z, \bar{S} \otimes \bar{E})$.

The remainder of this appendix is devoted to proving the following decay estimates for the resolvent of $H_{0,0}^{2}$. These estimates are needed in Sect. 5. For $\rho \in \mathbf{C}$ and $\lambda$ in $\mathbf{C}$, define

$$
H^{2}(\rho)=e^{-\rho y} H^{2} e^{\rho y}=H^{2}+2 \rho \theta\left(\frac{\partial}{\partial y}\right)+\rho^{2}: C_{0}^{\infty} \mapsto C_{0}^{\infty} .
$$


Proposition (A.6). Let $\lambda_{0}$ be a lower bound for the spectra of $\left(D_{\infty}\right)_{0,0}^{2}$ and $\left(D_{-\infty}\right)_{0,0}^{2}$. Let $\mathscr{F}$ be a closed set in $\mathbf{C}$ disjoint from the spectrum of $\mathrm{H}_{0,0}^{2}$ and contained in the half plane $\left\{\lambda: \operatorname{Re} \lambda<\lambda_{0}\right\}$. Then there exist constants $\rho_{0}>0$ and $c$ such that

1. For $|\rho|<\rho_{0}, \mathscr{F}$ is disjoint from the spectrum of $H_{0,0}^{2}(\rho)$, and for $\lambda \in \mathscr{F}$, the resolvent $\left(H_{0,0}^{2}(\rho)-\lambda\right)^{-1}$ is the closure on $L_{0}^{2}(\mathbf{R} \times X, \bar{S} \otimes \bar{E})$ of

$$
e^{-\rho y} R\left(H^{2}, \lambda\right) e^{\rho y}: C_{0}^{\infty} \mapsto C^{\infty} \text {. }
$$

2. For $|\rho|<\rho_{0}, \lambda \in \mathscr{F},\left|y-y^{\prime}\right|>1$, and $x, x^{\prime} \in X$,

$$
\left\|e^{-\rho y} R\left(H^{2}, \lambda\right) e^{\rho y}\right\|_{0,0}<c\left|\left(H_{0,0}^{2}-\lambda\right)^{-1}\left(y, x ; y^{\prime}, x^{\prime}\right)\right|<c e^{-\left|\rho \| y-y^{\prime}\right|} .
$$

3. If $H$ is invariant under translations of $Y$, then for $\rho, \lambda, y, y^{\prime}, x, x^{\prime}$ as in (2),

$$
\left|\left(H_{0,0}^{2}-\lambda\right)^{-1}\left(y, x ; y^{\prime}, x^{\prime}\right)\right|<c e^{-\left|\lambda-\lambda_{0}\right|^{1 / 2}\left|y-y^{\prime}\right|} .
$$

We remark that (A.6.1) is not immediate since it is not clear a priori that (A.7) extends to a bounded operator on $L^{2}$.

Proof. To prove (A.6.1), choose $\rho_{0}>0$ sufficiently small so that for $\lambda \in \mathscr{F}$ and $|\rho|<\rho_{0}$,

$$
\begin{gathered}
\operatorname{Re}\left(\lambda+|\rho|^{2}+|\rho|\right)<\lambda_{0}, \\
\left\|\left(H^{2}-\lambda\right)^{-1}\left(2 \rho \theta\left(\frac{\partial}{\partial y}\right)+\rho^{2}\right)\right\|<1 .
\end{gathered}
$$

From (A.8) and the usual appeal to the geometric series, it follows that for $|\rho|<\rho_{0}$ and $\lambda \in \mathscr{F}, H_{0,0}^{2}(\rho)-\lambda$ has a bounded inverse on $L^{2}(\mathbf{R} \times X, \bar{S} \otimes \bar{E})$. Now let $\psi_{0} \in C_{0}^{\infty}$ and set

$$
\psi=\left(H_{0,0}^{2}(\rho)-\lambda\right)^{-1} \psi_{0}-e^{-\rho y} R\left(H^{2}, \lambda\right) e^{\rho y} \psi_{0} .
$$

To complete the proof of (A.6.1) we must show that $\psi=0$. It is easy to see that $\psi$ is smooth and that $\left(H^{2}(\rho)-\lambda\right) \psi=0$. Since $\left(H_{0,0}^{2}(\rho)-\lambda\right)$ has an inverse as an unbounded operator on $L_{0}^{2}(\mathbf{R} \times X, \bar{S} \otimes \bar{E})$, it thus suffices to show that $\psi$ is in the domain of $H_{0,0}^{2}(\rho)$. For this it is enough to show that $\psi$ is exponentially decreasing in $|y|$, since then $\psi$ is in $L_{2}^{2}(\mathbf{R} \times X, \bar{S} \otimes \bar{E})$, and, as in Proposition (A.2), $L_{2}^{2}(\mathbf{R} \times$ $X, \bar{S} \otimes \bar{E})$ is contained in the domain of $H_{0,0}^{2}(\rho)$.

We will demonstrate the exponential decay for $y \rightarrow \infty$; the case $y \rightarrow-\infty$ is analogous. Let $\left\{\phi_{n}\right\}$ be an orthonormal basis for $L_{0}^{2}(X, S \otimes E)$ consisting of eigenfunctions of $\left(D_{\infty}\right)_{0,0}^{2}$ with eigenvalues $\left\{\lambda_{n}\right\}$ and set

$$
c_{n}(y)=\int_{X}|d x|\left\langle\phi_{n}(x), \psi(y, x)\right\rangle .
$$

For $y$ sufficiently large, $H^{2}(\rho)=-\partial^{2} / \partial y^{2}+D_{\infty}^{2}-\rho^{2}$, and so the equation $\left(H^{2}(\rho)-\lambda\right) \psi=0$ implies

$$
\left(\frac{\partial^{2}}{\partial y^{2}}+\rho^{2}-\lambda_{n}+\lambda\right) c_{n}(y)=0 .
$$

On the other hand, since $\left(H_{0,0}^{2}-\lambda\right)^{-1}$ and $\left(H_{0,0}^{2}(\rho)-\lambda\right)^{-1}$ are bounded operators on 
$L_{0}^{2}(\mathbf{R} \times X, \bar{S} \otimes \bar{E})$, it is clear from the definition (A.10) that $e^{-|\rho| y \mid} \psi$ is in $L^{2}(\mathbf{R} \times$ $X, \bar{S} \otimes \bar{E})$. Thus, $c_{n}(y)$ is in $L^{2}(\mathbf{R})$. Since condition (A.8) insures that the exponentially increasing solution of (A.12) can not be in $L^{2}(\mathbf{R})$, it follows that $e^{|\rho| l|y|} c_{n}(y)$ must be the exponentially decreasing solution, and then using (A.9) again it follows that $c_{n}(y)$ must also be exponentially decreasing. Thus $|\psi(y, x)|$ is exponentially decreasing in $y$ and the proof of (A.6.1) is complete.

Now the resolvent of $H_{0,0}^{2}(\rho)$ has $L_{0}^{2}(\mathbf{R} \times X, \bar{S} \otimes \bar{E})$ norm which is uniformly bounded for $\lambda \in \mathscr{F}$ and kernel which is continuous off the diagonal and uniformly bounded for $\lambda \in \mathscr{F}$ and $\left|y-y^{\prime}\right|>1$. (A.6.2) follows from this and (A.6.1).

Finally, (A.6.3) follows from the explicit expression, valid if $H$ is translation invariant, $\lambda \in \mathscr{F}$ and $\left|y-y^{\prime}\right|>0$,

$$
\left(H_{0,0}^{2}-\lambda\right)^{-1}\left(y, x ; y^{\prime}, x^{\prime}\right)=\sum_{n} \phi_{n}(x)\left\langle\phi_{n}\left(x^{\prime}\right), \cdot\right\rangle \frac{1}{2 \alpha_{n}} e^{-\left|y-y^{\prime}\right| \alpha_{n}} .
$$

Here $\left\{\phi_{n}\right\}$ is an orthonormal basis of eigenfunctions of $D_{0,0}^{2}$ with eigenvalues $\left\{\lambda_{n}\right\}$ and $\alpha_{n}^{2}=\lambda_{n}-\lambda$ with $\operatorname{Re} \alpha_{n}>0$. The estimate

$$
\sum e^{-\left|y-y^{\prime}\right| \lambda_{n} \mid}<\text { const } \sum|\lambda|^{-z}=\zeta\left(D^{2}\right)(z)<\infty, \quad \operatorname{Re} z>0
$$

implies that the sequence (A.13) is absolutely convergent, uniformly for $\left|y-y^{\prime}\right|>$ $\varepsilon>0$, and then standard arguments show that it actually gives the resolvent.

\section{B. A Generalization of Gilkey's Theorem}

In this Appendix we will give a generalization of the Gilkey Theorem ([3]). This was used in Sect. 2 and 3 to prove Lemmas 2.14 and 3.11.

For a manifold $X$, and a complex vector bundle $E$ over $W$ we consider regular form-valued invariants of a Riemannian metric $g$ on $X$, a connection $\nabla$ on $E$, and endomorphism valued differential forms $T_{j} \in \Omega(X$, End $(E)), j=1,2, \ldots, n$. To define what we mean by invariant we consider as in [3] the category $\mathscr{C}$ whose objects are manifolds $X^{\prime}$ and vector bundles $E^{\prime}$ over $X^{\prime}$, and whose morphisms are bundle maps $f: E^{\prime} \mapsto E^{\prime \prime}$ for which the map of base spaces $f: X^{\prime} \mapsto X^{\prime \prime}$ is a diffeomorphism onto an open submanifold. Then a differential form valued invariant of $g, \nabla$, and $T_{j}$ is a natural transformation $\tau$ from the functor

$$
\left.\mathscr{C} \mapsto\{\text { metrics on } Y \text {, connections on E, (endomorphism-valued forms on } Y)^{n}\right\}
$$

to the functor $\mathscr{C} \mapsto\{$ forms on $Y\}$. Thus $f^{*} \tau\left(g, \nabla, T_{1}, \ldots, T_{n}\right)=\tau\left(f^{*} g, f^{*} \nabla, f^{*} T_{1}, \ldots\right.$, $\left.f^{*} T_{n}\right)$. An invariant has weight $k$ if for any $\lambda>0, \tau\left(\lambda^{2} g, \nabla, T\right)=\lambda^{k} \tau(g, \nabla, T)$.

An invariant $\tau$ is called regular if for any local coordinate system $\chi:\left.E\right|_{U} \leftrightarrow \mathbf{R}^{\prime \prime \prime} \times \mathbf{C}^{l}$, $U \subset Y$, the components of $\tau\left(g, \nabla, T_{j}\right)$ are given by universal polynomials in $\operatorname{det}^{-1}\left(g_{\mu \nu}\right), g_{\mu v}, \nabla_{e \mu}^{d},\left(T_{j}\right)_{e \mu}^{d}$ and their derivatives. Here the components and derivatives of the various objects are taken relative to the coordinate system on $Y$ and trivialization of $E$ defined by $\chi$. The polynomials are universal in the sense that the same polynomial works for any choice of $\chi$.

The generalized Gilkey theorem which we need is 
Proposition (B.1). Any regular differential form valued invariant $\tau$ of weight zero is in the ring generated by the invariants

$$
\begin{aligned}
& \left(g, \nabla, T_{1}, \ldots, T_{n}\right) \mapsto \operatorname{tr}\left(\mathscr{R}_{g}\right)^{k}, \\
& \left(g, \nabla, T_{1}, \ldots, T_{n}\right) \mapsto \operatorname{tr} m\left(\mathscr{F}_{\nabla}, T_{1}, d_{\nabla} T_{1}, T_{2}, d_{\nabla} T_{2}, \ldots, T_{n}, d_{\nabla} T_{n}\right) .
\end{aligned}
$$

Here $\mathscr{R}_{g}$ is the curvature of the Levi-Civita connection for $g, \mathscr{F}_{\nabla}$ is the curvature of $\nabla$, and $d_{\nabla}$ is the covariant exterior defined by $\nabla . m$ is a monomial in $2+2 n$ variables and $\operatorname{tr} m(\cdots)$ is interpreted as the image of $m(\cdots)$ under

$$
\Omega^{*}\left(X,(\text { End } E)^{\otimes k}\right) \rightarrow \Omega^{*}(X, \text { End } E) \stackrel{\operatorname{tr}}{\longrightarrow} \Omega *(X),
$$

where the first arrow is induced by exterior product and composition in End $E$.

The proof is a straightforward generalization of the proof given in [3], in which the theorem without the $T_{j}$ is proved (see [8] for details).

\section{Traces of Operators Defined by Contour Integration}

In this Appendix we state a minor generalization of some results of Seeley [15] on operators of the form

$$
A(z)=\int_{\mathscr{C}} \frac{d \lambda}{2 \pi i} \lambda^{-z} R(\lambda)
$$

where $R(\lambda)$ is a suitable family of pseudo-differential operators. In particular we give formulae for local invariants obtained from the analytic continuation of $\operatorname{Tr} A(z)$. Seeley considered the case where $R(\lambda)$ is the resolvent of a single elliptic operator while we have in mind the case where $R(\lambda)$ is basically a product of resolvents, but this causes no essentially new difficulties.

Assumptions. We use the notation of Appendix D. Let $W$ be a smooth manifold of dimension $m$ and let $F \rightarrow W$ be a complex vector bundle with fiber of complex dimension $l$. Let $\mathscr{S}$ be an open set in $\mathbf{C}$ containing the negative real axis and the disk $\{\lambda:|\lambda|<1\}$. Let $R(\lambda), \lambda \in \mathscr{S}$, be a family of pseudo-differential operators acting on sections of $F$ and satisfying the following assumptions.

I. There exists a $c<0$ such that for $\lambda \in \mathscr{S}$ and $f \in C_{0}^{\infty},\|R(\lambda) f\|_{0}<c(1+|\lambda|)^{-1}\|f\|_{0}$.

II. $R(\lambda)$ can be approximated by pseudo-differential operators in the following sense. Suppose $\chi:\left.F\right|_{W} \mapsto \mathbf{R}^{m} \times \mathbf{C}^{l}$ is any coordinate trivialization for $U$ any open subset of $W$, and suppose $\phi, \psi \in C_{0}^{\infty}(U)$. Then there exists a $b(\lambda ; x, \xi) \in C^{\infty}(\mathscr{S} \times$ $\left.\mathbf{R}^{m} \times \mathbf{R}^{m}\right) \otimes \operatorname{End}\left(\mathbf{C}^{l}\right)$ satisfying the following conditions.

1. Let

$$
E_{\chi, \phi, \psi}(\lambda)=M_{\phi} \circ R(\lambda) \circ M_{\psi}-(\chi, \phi)^{*} \circ \mathrm{Op}(b(\lambda)) \circ(\chi, \psi)_{*} \cdot
$$

Then there are $c, \delta>0$ such that for $\lambda \in \mathscr{S}$ and $f \in C_{0}^{\infty}$,

$$
\left\|E_{\chi, \phi, \psi}(\lambda) f\right\|_{m / 2}<(1+|\lambda|)^{-1+\delta}\|f\|_{-m / 2}
$$

2. $b$ has an expansion of the form $b=b_{-1}+b_{-2}+\cdots+b_{-m-1}$ where $b_{n}(\lambda ; x, \xi) \in C^{\infty}\left(\mathscr{S} \times \mathbf{R}^{m} \times \mathbf{R}^{m}\right) \otimes \operatorname{End}\left(\mathbf{C}^{l}\right)$ satisfies 
a. For all multi-indices $\alpha$ and $\beta$

$$
\left|\frac{\partial^{\beta}}{\partial x^{\beta}} \frac{\partial^{\alpha}}{\partial \xi^{\alpha}} b_{n}(\lambda ; x, \xi)\right|<\operatorname{const}(1+|\xi|)^{n-|\alpha|} .
$$

b. For sufficiently large integers $j$ there exists a $c>0$ such that

$$
\left|\square_{\xi}^{j} b_{n}(\lambda ; x, \xi)\right|<\operatorname{const}(1+|\lambda|)^{-2}(1+|\xi|)^{4+n-2 j} .
$$

c. For $|\xi|^{2}+|\lambda|>1, b_{n}$ is analytic in $\lambda$ and homogeneous in $\left(\xi, \lambda^{1 / 2}\right)$ of degree $n$.

Here $\square_{\xi}=-\sum_{i} \partial^{2} / \partial \xi_{i}^{2}$. We have included in I an assumption about the decay of $\|R(\lambda)\|_{0,0}$ since, on a non-compact manifold, information about the global $L^{2}$ norm does not follow from the local assumptions in II. Note that (C.3) implies that $E_{\chi, \phi, \psi}(\lambda)$ has a continuous kernal $E_{x, \phi, \psi}\left(\lambda ; x, x^{\prime}\right)$ with

$$
\left|X_{\chi, \phi, \psi}\left(\lambda ; x, x^{\prime}\right)\right|<c(1+|\lambda|)^{-1+\delta} .
$$

The somewhat unwieldy conditions I and II are satisfied quite naturally for the families of operators $R(\lambda)$ which arise in connection with resolvents of differential operators. In particular they are satisfied in all the situations we consider in the body of the paper.

Let $\mathscr{C}$ be the clockwise oriented curve in $\mathscr{S}$ which goes from $-\infty$ to -1 along the ray $\{\arg \lambda=\pi\}$, then clockwise around the circle $|\lambda|=1$, and then from -1 to $-\infty$ on the ray $\{\arg \lambda=-\pi\}$. Let $\mathcal{O}$ be the counterclockwise oriented circle $|\lambda|=1$. Define

$$
A(z)=\int_{\mathscr{C}} \frac{d \lambda}{2 \pi i} \lambda^{-z} R(\lambda), \quad P=\int_{\mathcal{O}} \frac{d \lambda}{2 \pi i} R(\lambda),
$$

with the complex powers $\lambda^{-z}$ defined using the branch of the logarithm with cut along the negative real axis and $\log (1)=0$. By assumption $\mathrm{I}, A(z)$ and $P$ define bounded operators on $L^{2}$.

Results. Let $\chi, U, \phi, \psi$, and $b_{n}$ be as in II.2 above. Let $P_{\chi, \phi, \psi}\left(x, x^{\prime}\right)$ and $A_{\chi, \phi, \psi}\left(z ; x, x^{\prime}\right)$ for $\operatorname{Re} z>0$ denote the kernels of $P$ and $A$ relative to $\{\chi, \phi, \psi\}$. The main result of this Appendix is

\section{Proposition (C.6).}

1. For $\operatorname{Re} z>(m+1) / 2, A_{x, \phi, \psi}\left(z ; x, x^{\prime}\right)$ is analytic in $z$ and continuous in $x, x^{\prime}$.

2. $A_{\chi, \phi, \psi}(z ; x, x)$ extends to a meromorphic function of $z$ for $\operatorname{Re} z>-1$ which is continuous in $x$. The only singularities are possible simple poles at half integer values of $z$, and there is no pole at $z=0$.

3. For sufficiently large $j$,

$$
\begin{aligned}
\operatorname{Re} & s_{\{z= \pm 1 / 2\}} A_{x, \phi, \psi}(z ; x, x) \\
& =\frac{1}{2(2 \pi)^{m}} \frac{(m-1) !}{(m-1+2 j) !} \int_{|\xi|=1} d^{m-1} \xi \int_{0}^{\infty} d t \square_{\xi}^{j} b_{-m \pm 1}(-t ; x, \xi) .
\end{aligned}
$$


4. If $P_{\chi, \phi, \psi}\left(x, x^{\prime}\right)$ is continuous in $x$ and $x^{\prime}$, then for sufficiently large $j$,

$$
\begin{aligned}
& \lim _{z \rightarrow 0} A_{\chi, \phi, \psi}(z ; x, x)+P_{\chi, \phi, \psi}(x, x) \\
& \quad=\frac{1}{2\left(2 \pi^{m}\right)} \frac{(m-1) !}{(m-1+2 j) !} \int_{|\xi|=1} d^{m-1} \xi \int_{0}^{\infty} d t \square_{\xi}^{j} b_{-m-2}(-t ; x, \xi) .
\end{aligned}
$$

Here $d^{m-1} \xi$ denotes the standard volume form on the $m-1$ sphere $|\xi|=1$.

It is statement (C.6.4) that is not immediately contained in the work of Seeley. For a proof of (C.6), see [8].

Proposition (C.6) expresses the residues at the poles of $A_{\chi, \phi, \psi}(z ; x, x)$ and the value $\lim _{z \rightarrow 0} A_{\chi, \phi, \psi}(z ; x, x)$ in terms of integrals of particular homogeneous terms in the symbol expansion for $R(\lambda)$. If we restrict the form of the symbol expansion we can perform these integrals to obtain more detailed information.

Specifically, assume that in addition to II.2.a and II.2.b, the functions $b_{n}(\lambda ; x, \xi)$ satisfy

II.2.c For $|\xi|^{2}+|\lambda|>1$,

$$
\operatorname{tr} b_{n}(\lambda ; x, \xi)=\sum_{q, r} c_{n, q, r}(x, \xi) \lambda^{q}(a(x, \xi)-\lambda)^{-r},
$$

where $c_{n, q, r}(x, \xi) \in C^{\infty}\left(\mathbf{R}^{m}, \mathbf{R}^{m}\right) \otimes \operatorname{End}\left(\mathbf{C}^{l}\right)$ is a polynomial function of degree $n-2 q$ $+2 r$ in $\xi$ and for all $x, a(x, \xi) \in C^{\infty}\left(\mathbf{R}^{m} \times \mathbf{R}^{m}\right)$ is a strictly positive quadratic form in $\xi$.

Condition II.2.c will be satisfied if $R(\lambda)$ is the product of local operators and the resolvents of second order differential operators with the same positive definite scalar leading symbol. The families $R(\lambda)$ considered in this paper are all of this type.

Following [3] we deduce

Corollary (C.8). Under the additional assumption II.c, $\operatorname{Re} s_{\{s= \pm 1 / 2\}} A_{\chi, \phi, \psi}(z ; x, x)$ and $\lim _{z \rightarrow 0} A_{\chi, \phi, \psi}(z ; x, x)$ depends polynomially on the coefficients of the $c_{n, q, r}(x, \xi)$, the coefficients of $a(x, \xi)$, and the function $\operatorname{det}^{-1} a(x)$.

For a proof of Corollary (C.8,) see [8].

\section{Notational Conventions}

Let $W=X$ and $F=S \otimes E$, or $W=\mathbf{R} \times X$ and $F=\bar{S} \otimes \bar{E}$. Put a metric on $W$ and a fiberwise Hermetian inner product and compatible connection on $F$. For $W=$ $\mathbf{R} \times X$ we impose the additional requirement that the connections and inner products be invariant under translations in the $\mathbf{R}$ direction. This is a reasonable condition since we are assuming (see condition 1 of Sect. 1) that our operators $H$ are translation invariant for large $|y|, y \in \mathbf{R}$.

For integer $k$, let $\|\cdot\|_{k}$ denote the Sobolev norms on $C_{0}^{\infty}(W, F)$, and let $L_{k}^{2}(W, F)$ be the Sobolev spaces obtained by completing $C_{0}^{\infty}(W, F)$ relative to these norms. For a linear operator $A: C_{0}^{\infty}(W, F) \mapsto L_{j}^{2}(W, F)$, let $A_{j, k}: L_{k}^{2}(W, F) \rightarrow L_{j}^{2}(W, F)$ denote its (possibly unbounded) closure, if this closure exists. (Recall that the graph of the closure of $A$ in $L_{k}^{2}(W, F) \times L_{j}^{2}(W, F)$ is the closure of the graph of $A$.) We will often omit the subscripts $j, k$ if $j=k=0$. 
In Appendix $\mathrm{C}$ we also use the following notation. For $\chi:\left.E\right|_{U} \mapsto \mathbf{R}^{m} \times \mathbf{C}^{l}$ a local trivialization over some coordinate patch $U$ in $W$, and $\phi, \quad \psi \in C_{0}^{\infty}(U), \quad$ let $(\chi, \phi)^{*}=\chi^{*} \circ M_{\chi * \phi}: C^{\infty}\left(\mathbf{R}^{m}\right) \otimes \mathbf{C}^{l} \mapsto C_{0}^{\infty}(W, F)$ and $(\chi, \phi)_{*}=$ $\chi_{*} \circ M_{\phi}: C^{\infty}(W, F) \mapsto C_{0}^{\infty}\left(\mathbf{R}^{m}\right) \otimes \mathbf{C}^{l}$, where $M_{\phi}$ denotes multiplication by $\phi$. For a linear operator $A: C_{0}^{\infty}(W, F) \mapsto C^{\infty}(W, F)$, let $A_{\chi, \phi, \psi}=(\chi, \phi)_{*} \circ A \circ(\chi, \psi)^{*}$. Also, following [15], for $b \in C^{\infty}\left(\mathbf{R}^{m} \times \mathbf{R}^{m}\right) \otimes \operatorname{End}\left(\mathbf{C}^{l}\right)$, let $\mathrm{Op}(b): C_{0}^{\infty}\left(\mathbf{R}^{m}\right) \otimes \mathbf{C}^{l} \mapsto C^{\infty}\left(\mathbf{R}^{m}\right) \otimes \mathbf{C}^{l}$ be the pseudodifferential operator

$$
\mathrm{Op}(b) f(x)=(2 \pi)^{-m} \int d^{m} \xi e^{i x \xi} b(x, \xi) \int d^{m} y e^{i y \xi} f(y),
$$

when the integrals, in the order given, are defined.

Acknowledgements. We wish to thank L. Alvarez-Gaumé for his collaboration on part of this work [2]. We also wish to thank R. Bott, M. Goulian, G. Moore, P. Nelson, and C. Taubes for valuable discussions.

\section{References}

1. Alvarez-Gaumé, L., Della Pietra, S., Della Pietra, V.: The effective action for Chiral fermions. Phys. Lett. B. (to appear)

2. Alvarez-Gaumé, L., Della Pietra, S., Della Pietra, V.: The determinant of the Chiral Dirac operator and the eta invariant, Commun. Math Phys. (in press)

3. Atiyah, M. F., Bott, R., Patodi, V. K.: On the heat equation and the index theorem. Invent. Math. 19, 279 (1975)

4. Atiyah, M. F., Patodi, V. K., Singer, I. M.: Spectral asymmetry and Riemannian geometry I, II, III. Math. Proc. Camb. Phil. Soc. 77, 43 (1975), 78, 405 (1975), 79, 71 (1976)

5. Atiyah, M. F., Singer, I. M.: Dirac operators coupled to vector potentials. Proc. Nat'l. Acad. Sci. 81, 2597 (1984)

6. Bismut, J.-M., Freed, D.: Geometry of elliptic families: Anomalies and determinants, preprint

7. Bismut, J.-M., Freed, D.: The analysis of elliptic families: Metrices and connections on determinant bundles and the analysis of elliptic families: Dirac operators, eta invariants, and the holonomy theorem. Commun. Math. Phys. 106, 159 (1986) and 107103 (1986)

8. Della Pietra, V.: Ph.D Thesis, Harvard University, 1987 (unpublished)

9. Della Pietra, S., Della Pietra, V.: Parallel transport in the determinant line bundle: The non-zero index case. Commun. Math. Phys. (in press)

10. Gilkey, P. B., Smith, L.: The eta invariant for a class of elliptic boundary value problems. Commun. Pure Appl. Math. 36, 85 (1983)

11. Kato, T.: Perturbation theory for linear operators. Berlin, Heidelberg, New York: Springer 1980

12. Lott, J.: Vacuum charge and the eta function. Commun. Math. Phys. 93, 533 (1984)

13. Quillen, D.: Determinants of Cauchy Riemann operators over a Riemann surface, preprint

14. Reed, M., Simon, B.: Methods of modern mathematical physics, Vol. I., II., IV. New York, London: Academic Press 1978

15. Seeley, R.: Complex powers of an elliptic operators. In: Singular integrals. Proc. Sym. Pure Math. Vol. X, Am. Math. Soc. 1967

16. Seeley, R.: Topics in pseudo differential operators. 1968 CIME Lectures. In: Pseudo differential operators, Rome: Edizioni Cremonese 1969

17. Witten, E.: An $S U(2)$ anomaly, Phys. Lett. 117B, 324 (1982)

18. Witten, E.: Global graviational anomalies. Commun. Math. Phys. 100, 197 (1985)

Communicated by A. Jaffe

Received September 22, 1986; in revised form December 22, 1986 
